\title{
Stereocontrolled Preparation of Diversely Tri-Functionalized Cyclobutanes
}

Zong Chang, Régis Guillot, Thomas Boddaert,* and David J. Aitken*

CP3A Organic Synthesis Group \& Services Communs, ICMMO, CNRS UMR 8182, Université Paris Sud, Université Paris Saclay, 15 rue Georges Clemenceau, 91405 Orsay Cedex, France.

david.aitken@u-psud.fr, thomas.boddaert@u-psud.fr

\section{Supporting Information}

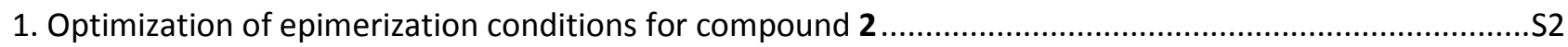

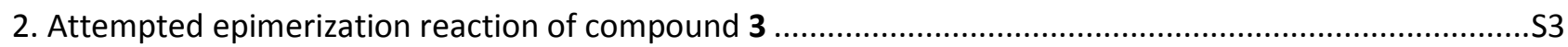

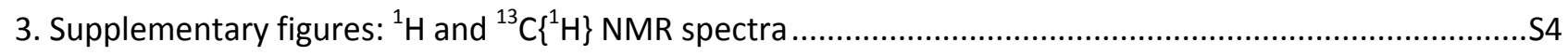

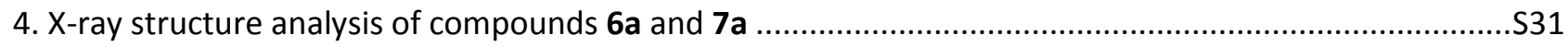




\section{Optimization of epimerization conditions for compound 2}

Table S1. Optimization of epimerization conditions.

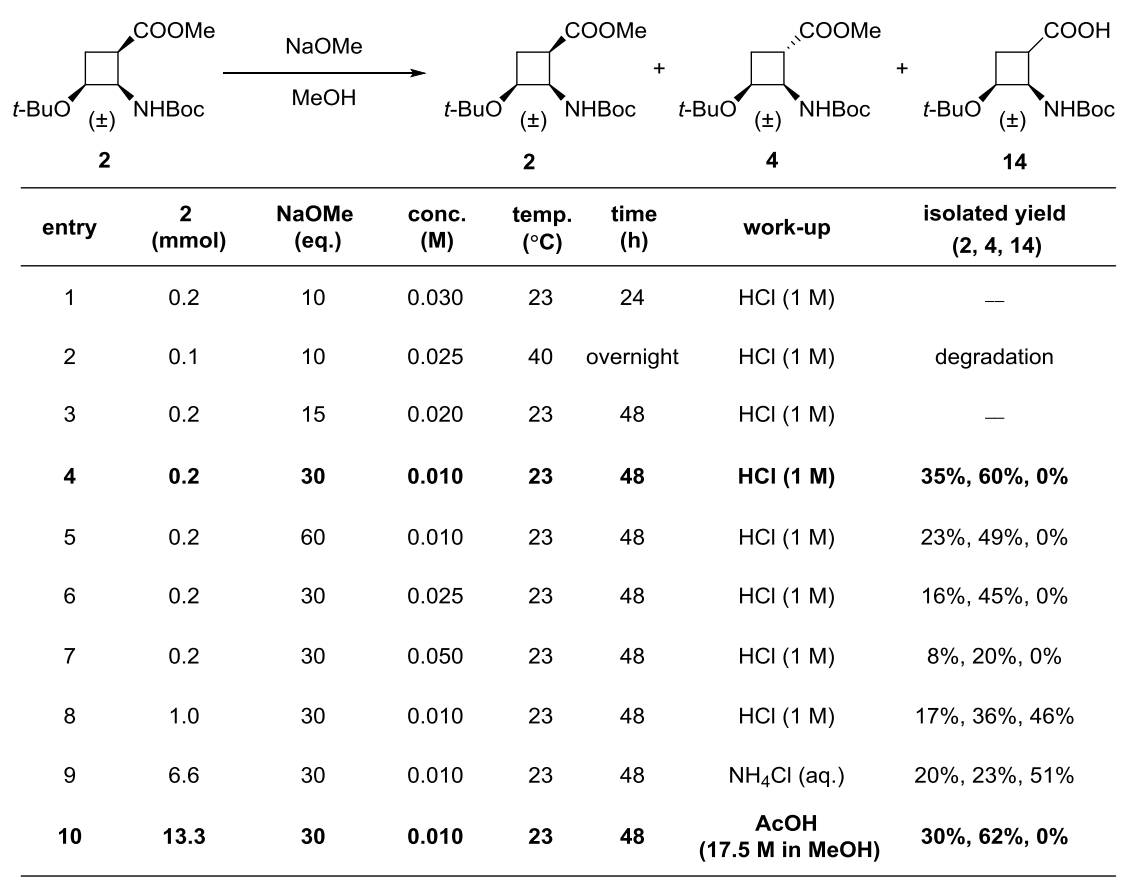




\section{Attempted epimerization reaction of compound 3}<smiles>CC(=O)OC(C)C1CC(O)C1NC(C)(C)C</smiles>

$( \pm)$

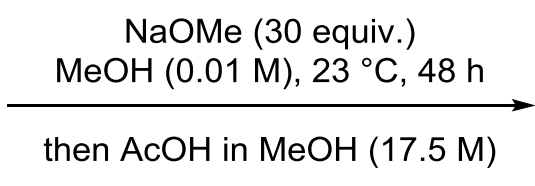

then $\mathrm{AcOH}$ in $\mathrm{MeOH}(17.5 \mathrm{M})$<smiles>COC(=O)NC1[C@H](O)C[C@H]1OC(C)(C)C</smiles>

$( \pm)$

3

Scheme S1. Epimerization reaction of compound $\mathbf{3}$

(士)-trans-cis-Methyl 2-((tert-butoxycarbonyl)amino)-3-hydroxycyclobutane-1-carboxylate 5 . To a freshly prepared $\mathrm{NaOMe}$ solution in methanol $(0.4 \mathrm{M}, 26 \mathrm{~mL})$, a solution of compound $3(100 \mathrm{mg}, 0.41 \mathrm{mmol})$ in methanol $(4 \mathrm{~mL})$ was added at $0{ }^{\circ} \mathrm{C}$. The mixture was stirred at room temperature for $48 \mathrm{~h}$. The reaction mixture was then poured into cold $\left(0^{\circ} \mathrm{C}\right)$ mixture of acetic acid $(4 \mathrm{~mL})$ and dry methanol $(4 \mathrm{~mL})$. After evaporation of the solvents under reduced pressure, the residue was diluted with $\mathrm{H}_{2} \mathrm{O}$ and extracted with $\mathrm{Et}_{2} \mathrm{O}$. The combined organic phases was washed with $\mathrm{H}_{2} \mathrm{O}$, brine and was dried over $\mathrm{MgSO}_{4}$ and evaporated. The residue was purified by flash chromatography (EtOAc/petroleum ether, 30/70 to 40/60) to afford $45 \mathrm{mg}$ of an inseparable mixture of compounds containing derivative $\mathbf{5}$ (red spots).

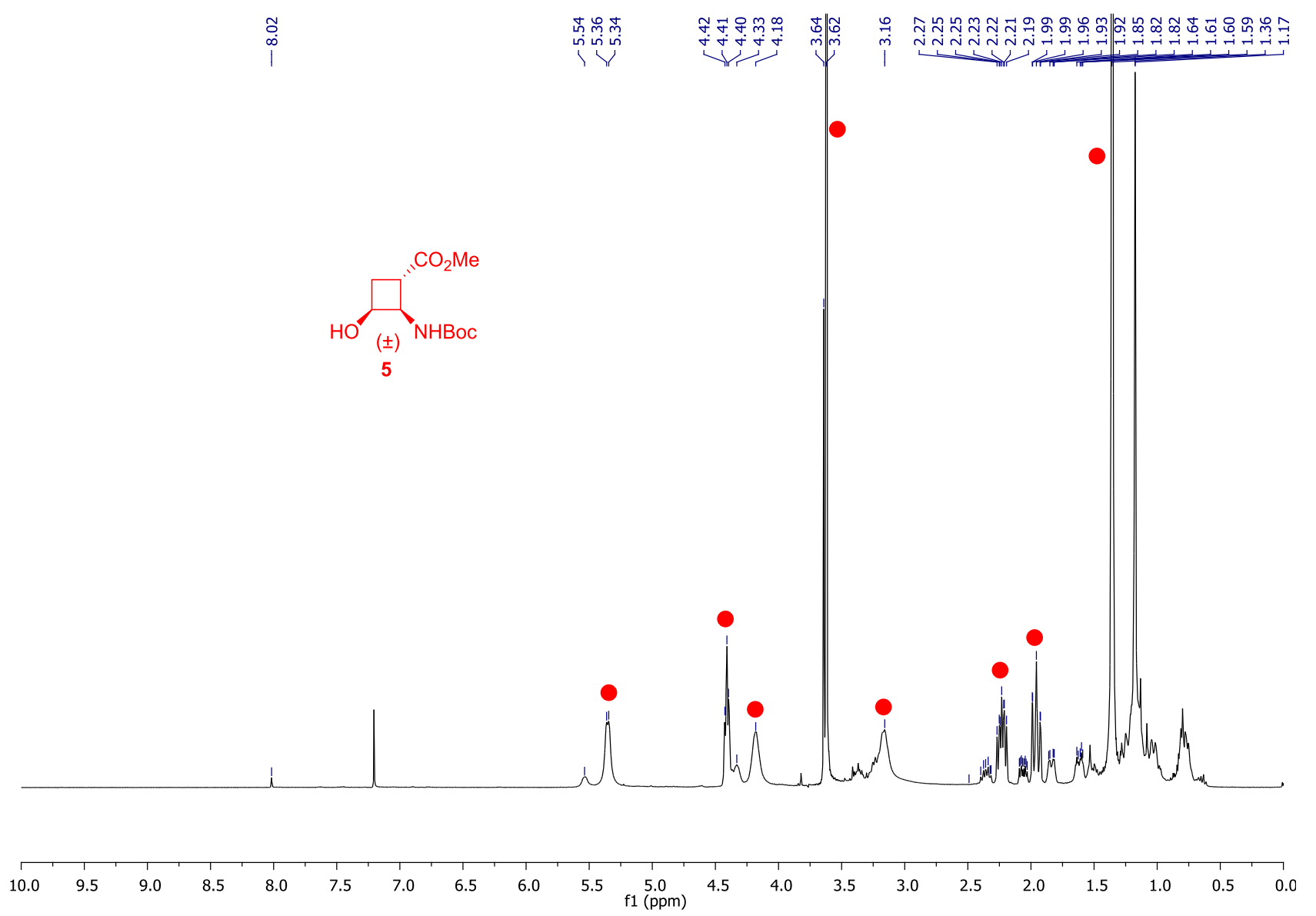


3. Supplementary figures: ${ }^{1} \mathrm{H}$ and ${ }^{13} \mathrm{C}\left\{{ }^{1} \mathrm{H}\right\}$ NMR spectra

(士)-cis-cis-Methyl 3-(tert-butoxy)-2-((tert-butoxycarbonyl)amino)cyclobutane-1-carboxylate 2:
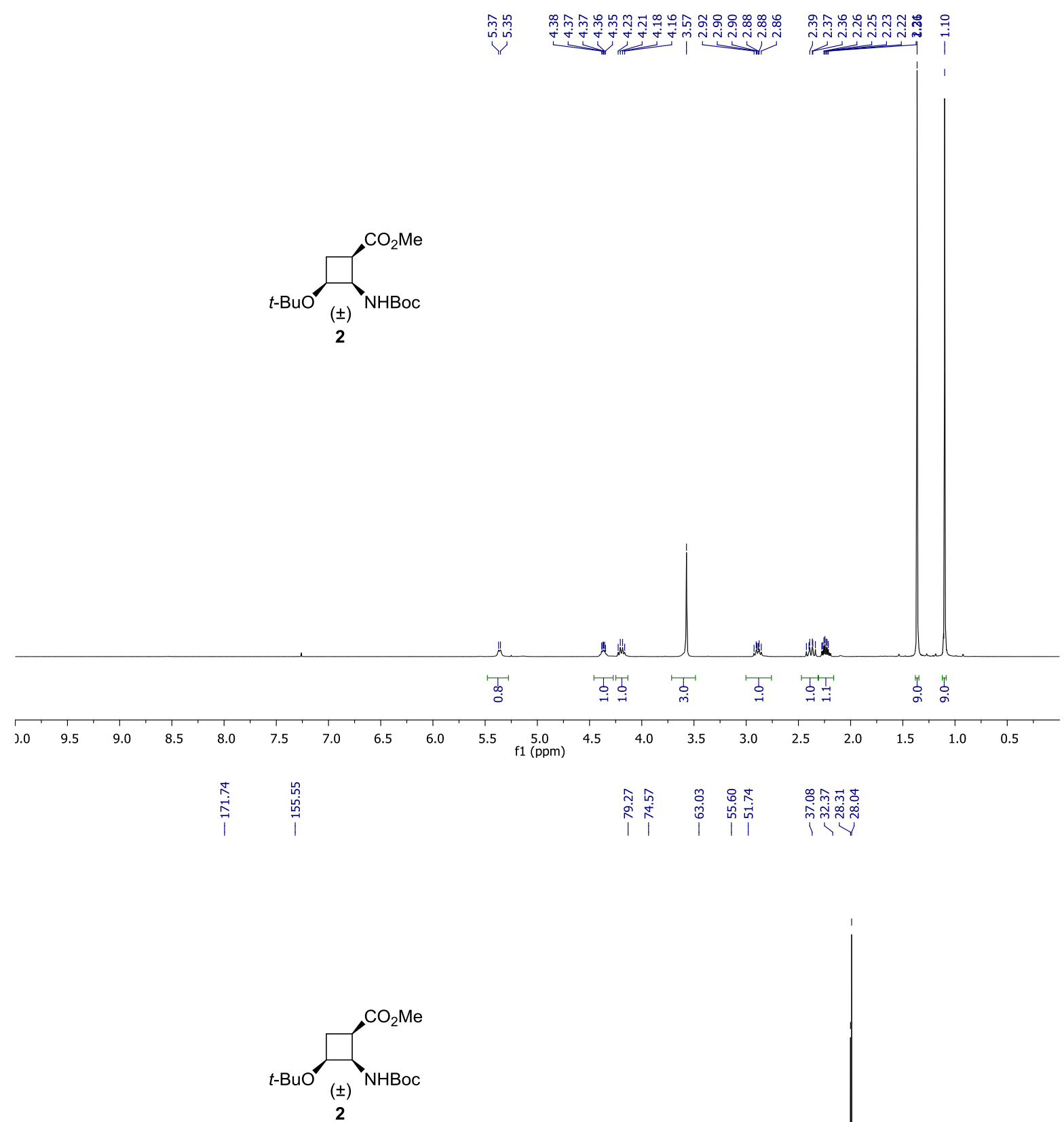

$\begin{array}{lllllllllllllllllllllll}210 & 200 & 190 & 180 & 170 & 160 & 150 & 140 & 130 & 120 & 110 & 100 & 90 & 80 & 70 & 60 & 50 & 40 & 30 & 20 & 10 & 0 & -10\end{array}$ 
(士)-cis-cis-Methyl 2- ((tert-butoxycarbonyl)amino)-3-hydroxycyclobutane-1-carboxylate 3:

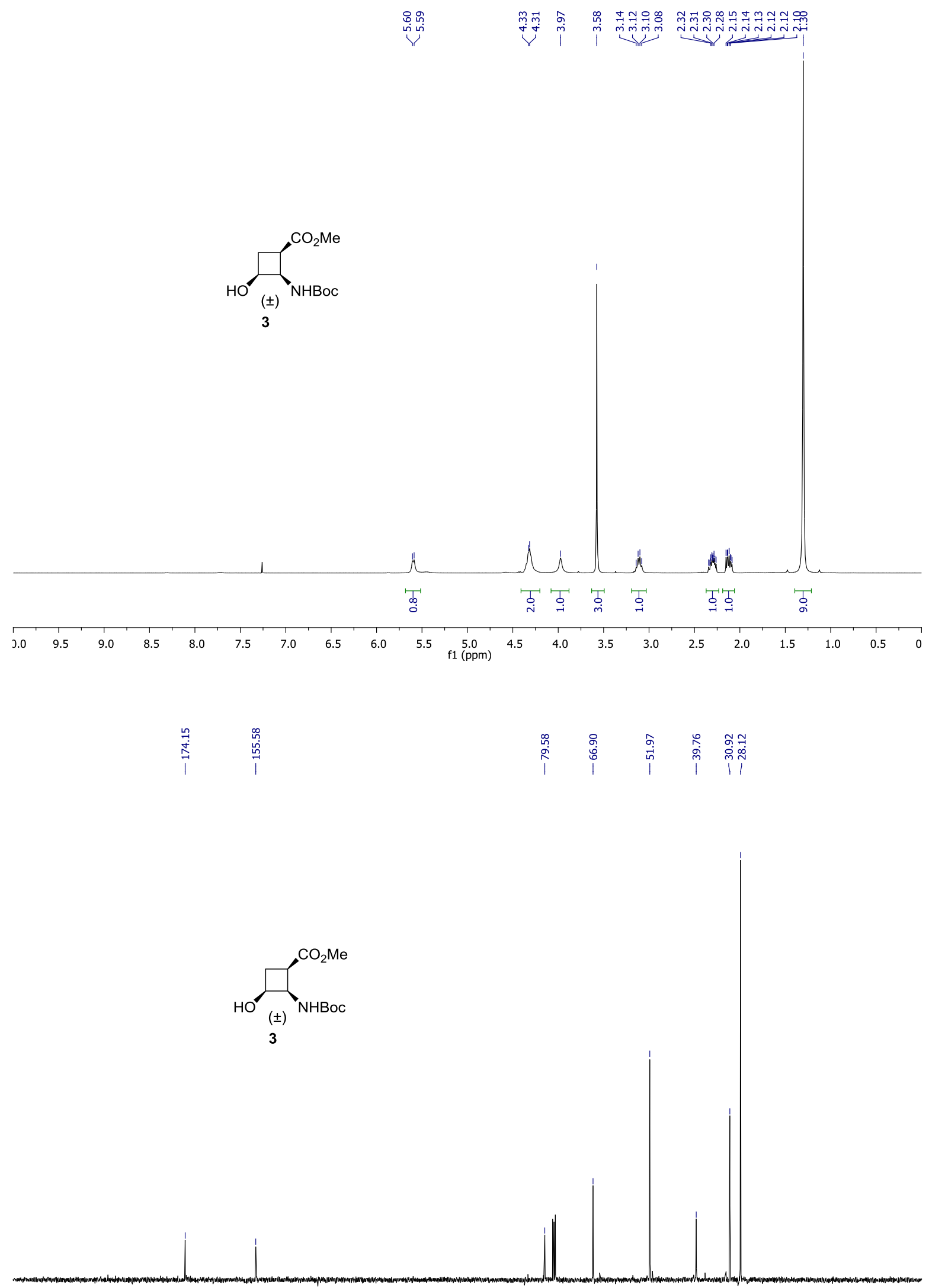

$\begin{array}{llllllllllllllllllllllllll}210 & 200 & 190 & 180 & 170 & 160 & 150 & 140 & 130 & 120 & 110 & 100 & 90 & 80 & 70 & 60 & 50 & 40 & 30 & 20 & 10 & 0 & -10\end{array}$ 
( \pm )-trans-cis-Methyl 3-(tert-butoxy)-2-((tert-butoxycarbonyl)amino)cyclobutane-1-carboxylate 4:
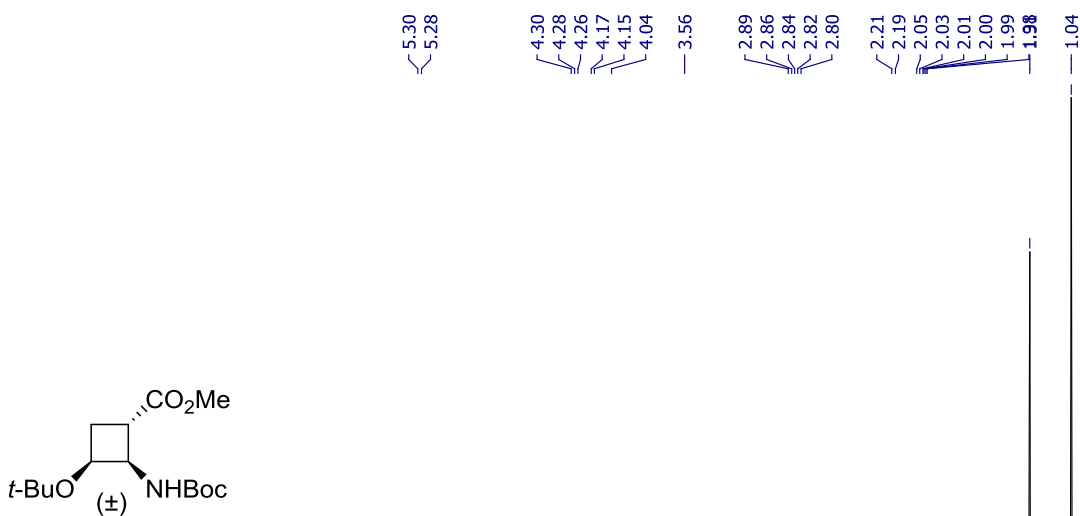

4
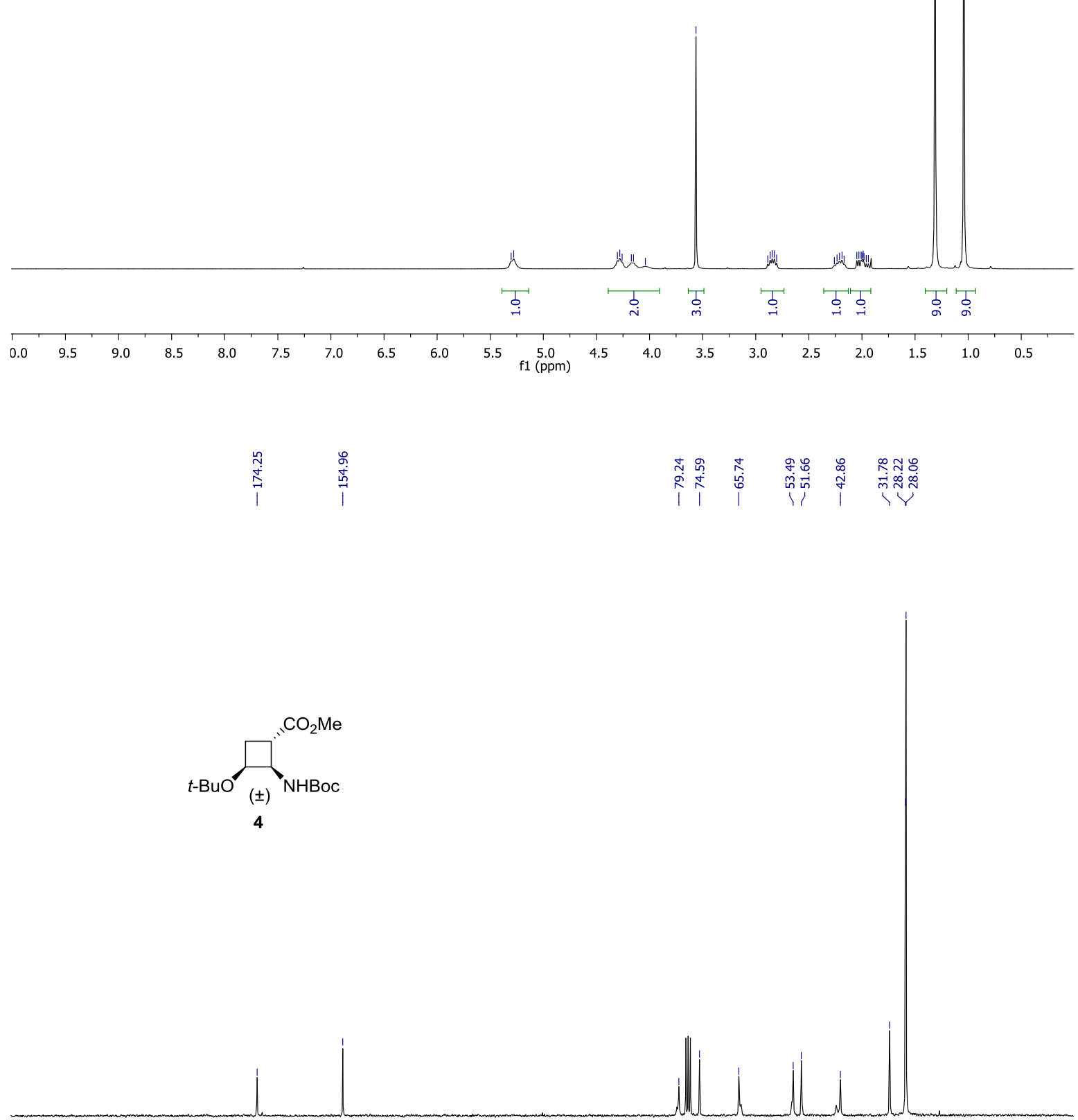

$\begin{array}{lllllllllllllllllllllll}220 & 210 & 200 & 190 & 180 & 170 & 160 & 150 & 140 & 130 & 120 & \begin{array}{c}110 \\ \mathrm{f} 1(\mathrm{ppm})\end{array} & 100 & 90 & 80 & 70 & 60 & 50 & 40 & 30 & 20 & 10 & 0\end{array}$ 
(士)-trans-cis-Methyl 2-((tert-butoxycarbonyl)amino)-3-hydroxycyclobutane-1-carboxylate 5:

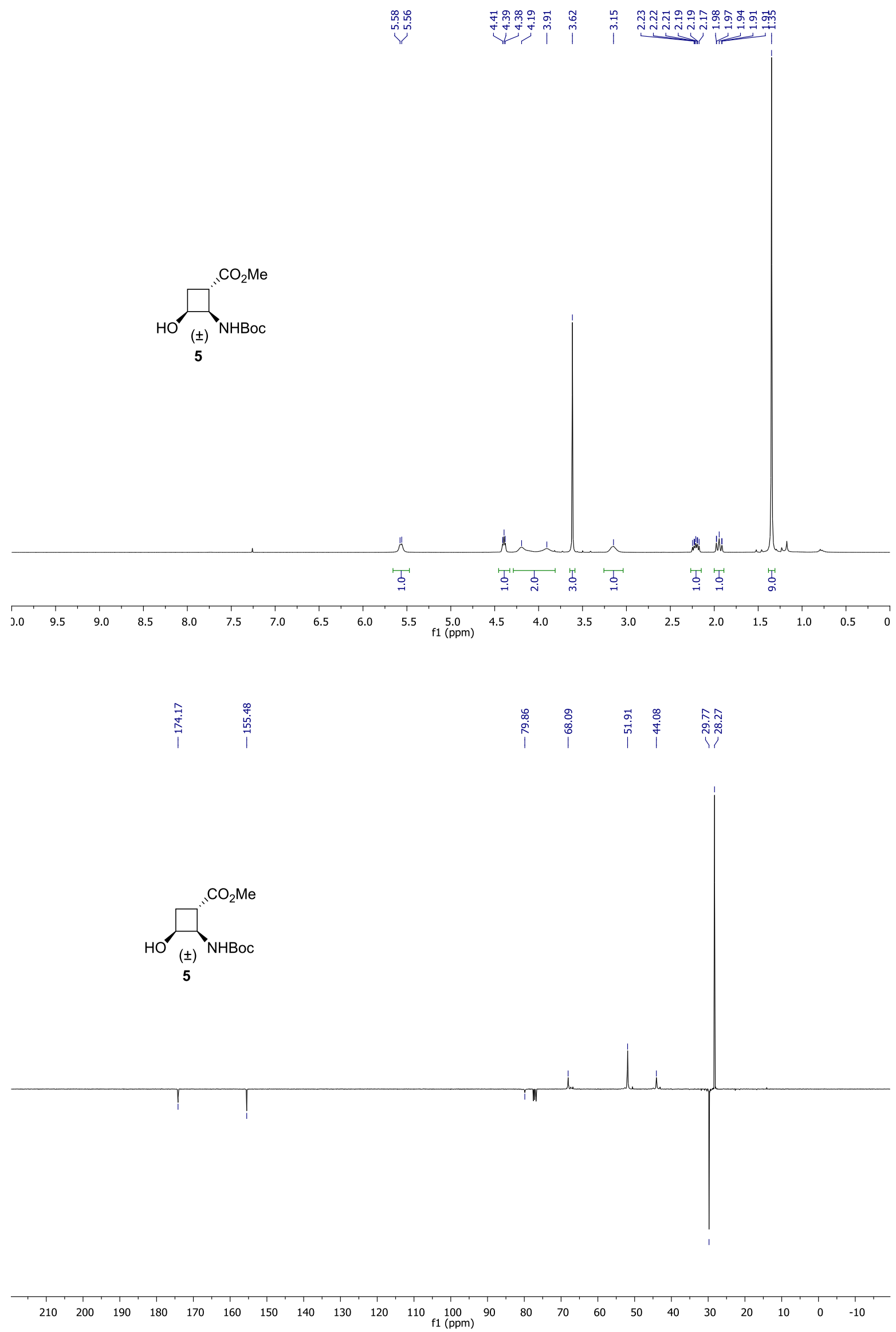


(士)-cis-trans-2-((tert-Butoxycarbonyl)amino)-3-(methoxycarbonyl)cyclobutyl 4-nitrobenzoate 6a:
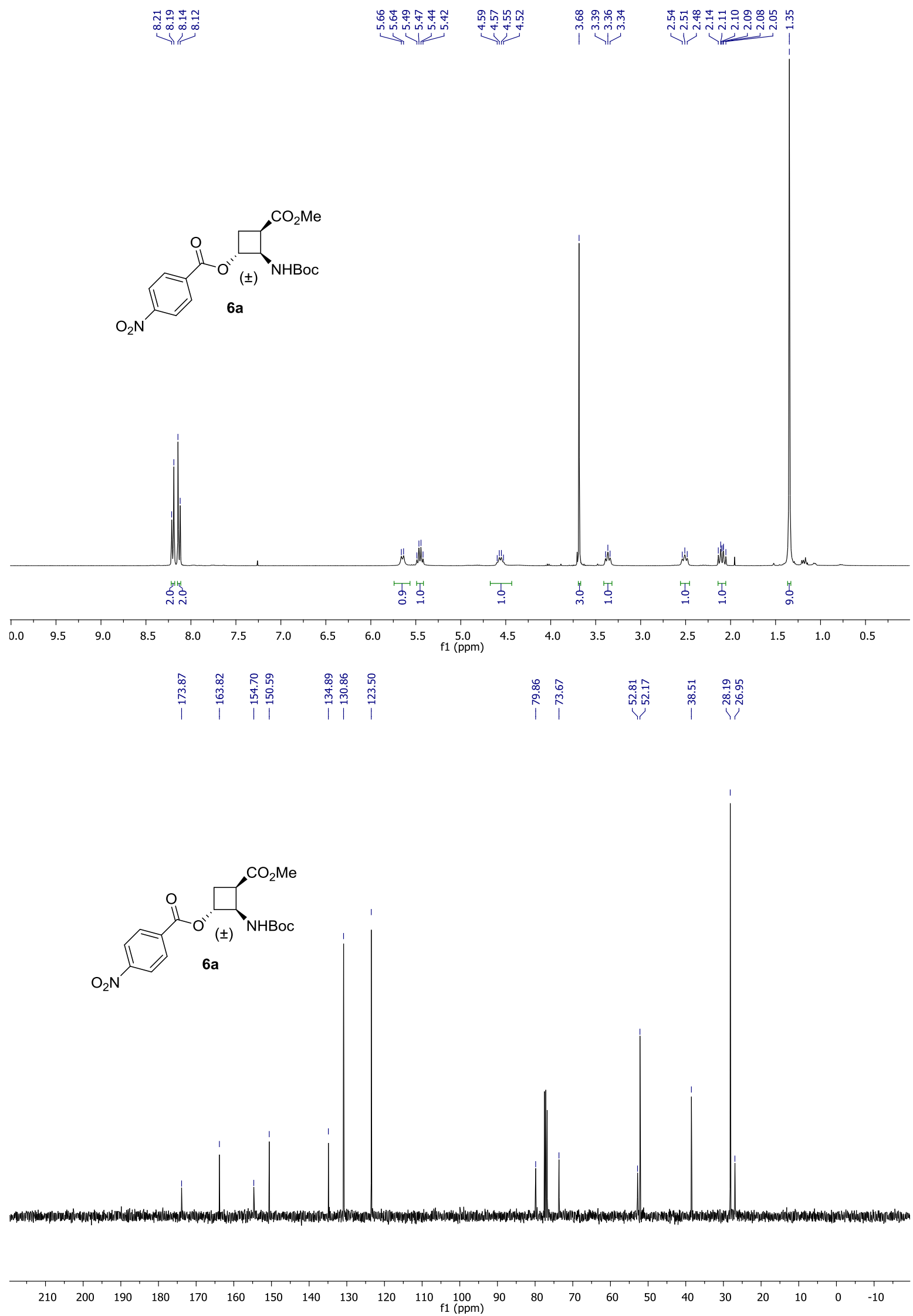
( \pm )-trans-trans-2-((tert-Butoxycarbonyl)amino)-3-(methoxycarbonyl)cyclobutyl 4-nitrobenzoate 7a:

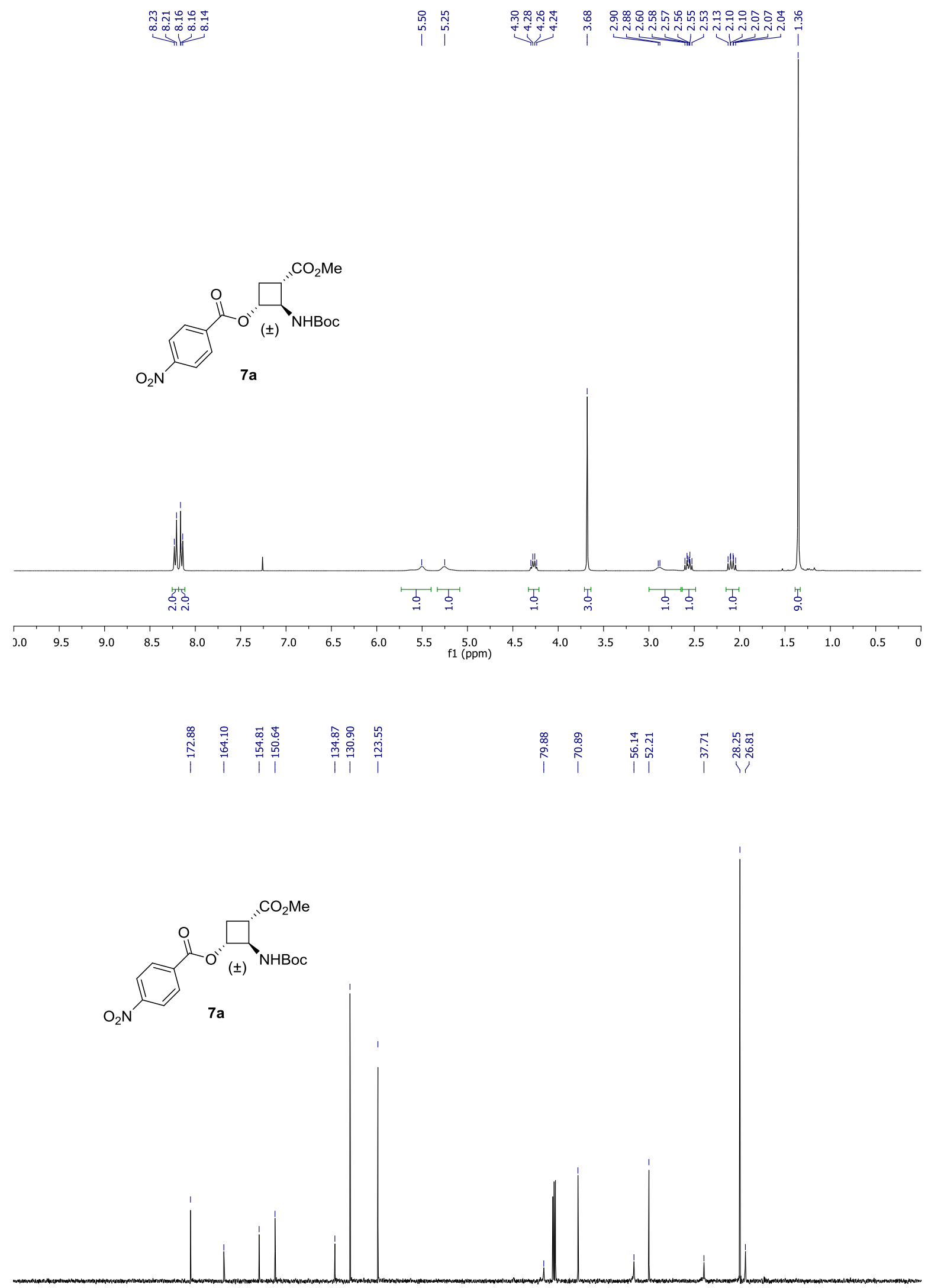

$\begin{array}{lllllllllllllllllllllllllll}210 & 200 & 190 & 180 & 170 & 160 & 150 & 140 & 130 & 120 & 110 & 100 & 100 & 90 & 70 & 60 & 50 & 40 & 30 & 20 & 10 & 0 & -10\end{array}$ 
(士)-cis-trans-Methyl 3-(benzoylthio)-2-((tert-butoxycarbonyl)amino)cyclobutane-1-carboxylate 6b:

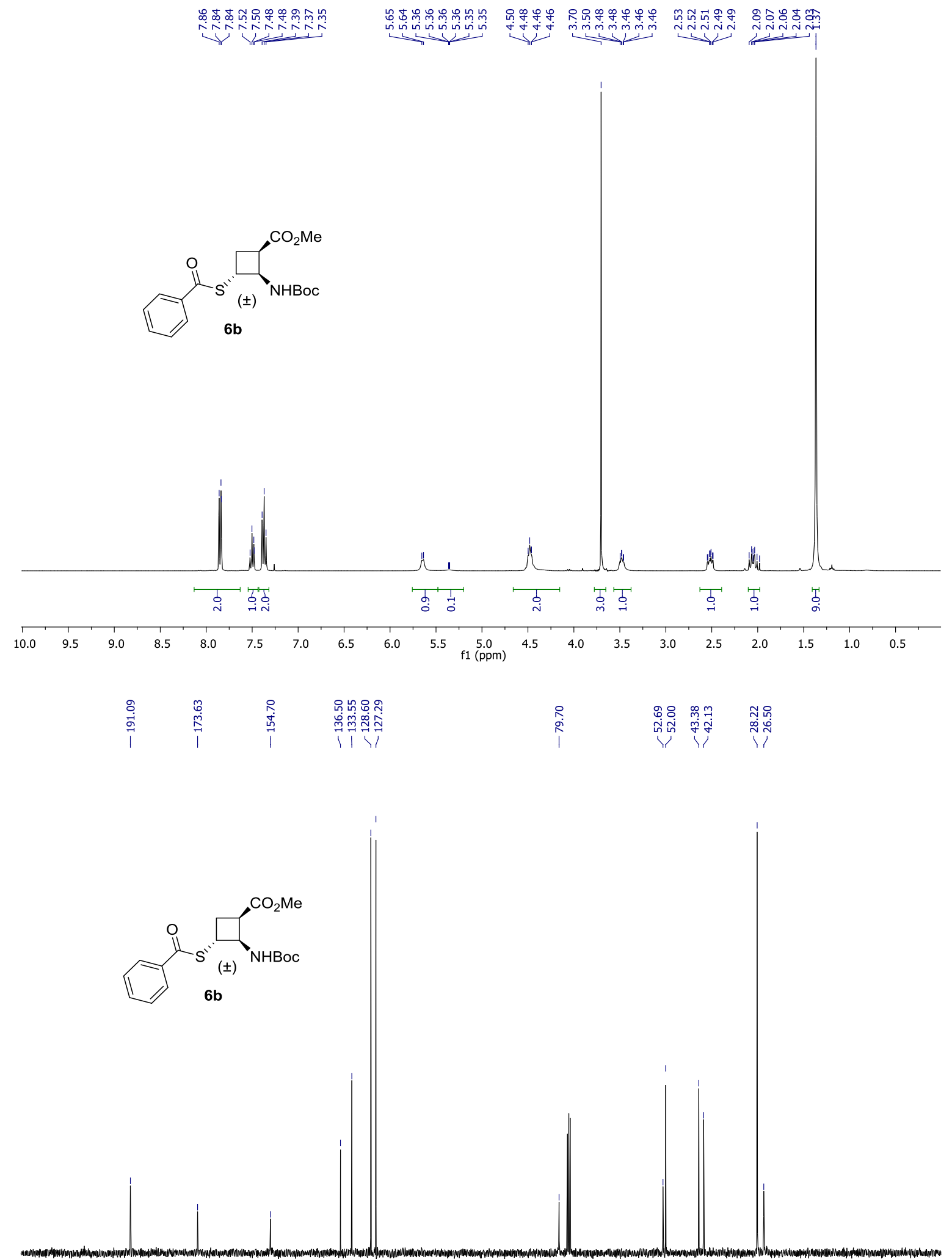

$\begin{array}{lllllllllllllllllllllllllll}210 & 200 & 190 & 180 & 170 & 160 & 150 & 140 & 130 & 120 & 110 & \begin{array}{c}100 \\ \mathrm{f} 1(\mathrm{ppm})\end{array} & 90 & 80 & 70 & 60 & 50 & 40 & 30 & 20 & 10 & 0 & -10\end{array}$ 
(士)-trans-trans-Methyl 3-(benzoylthio)-2-((tert-butoxycarbonyl)amino)cyclobutane-1-carboxylate 7b:

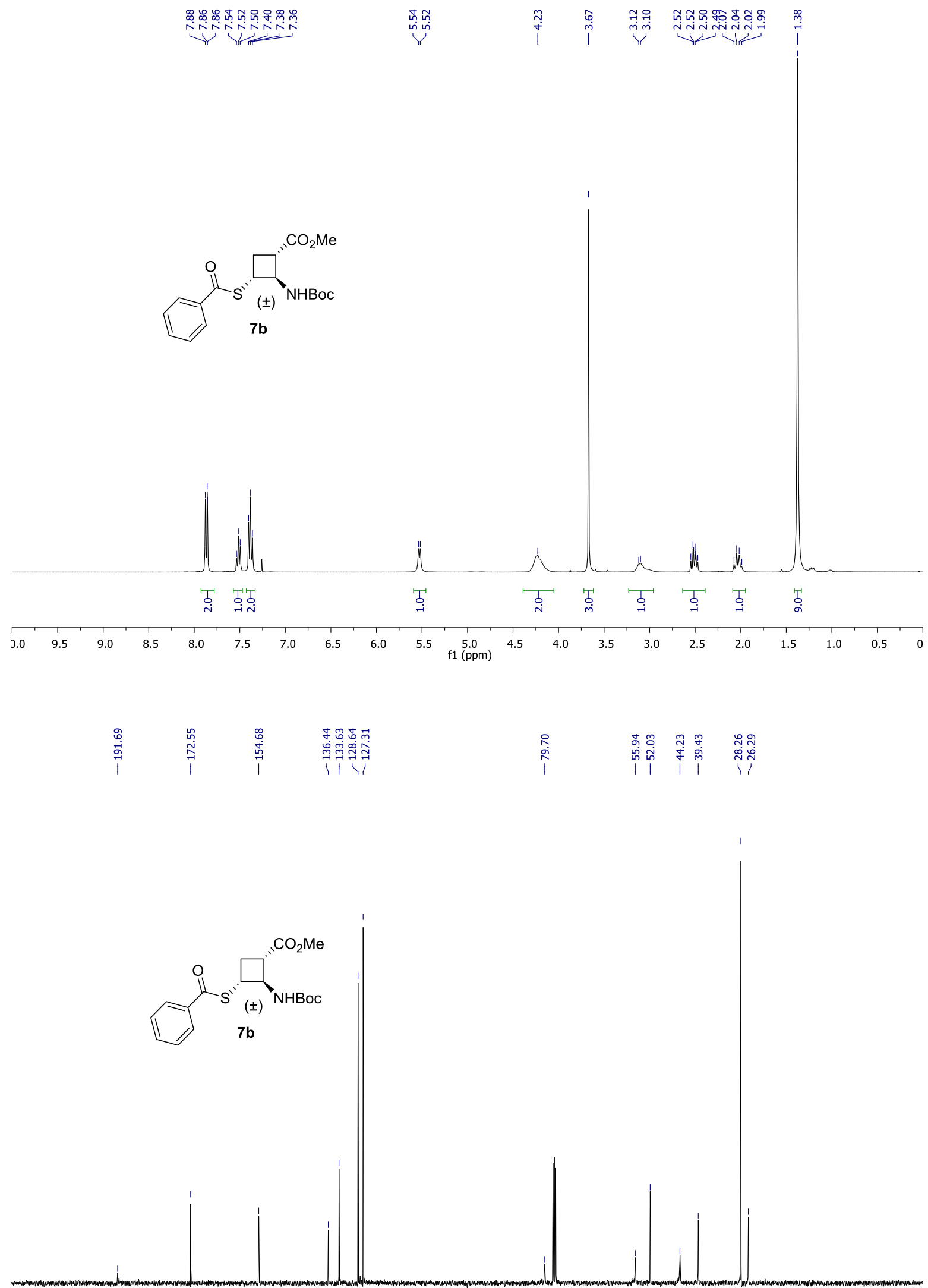

$\begin{array}{lllllllllllllllllllllllllll}210 & 200 & 190 & 180 & 170 & 160 & 150 & 140 & 130 & 120 & 110 & 100 & 100 & 80 & 70 & 60 & 50 & 40 & 30 & 20 & 10 & 0 & -10\end{array}$ 
( \pm )-cis-trans-Methyl 3-azido-2-((tert-butoxycarbonyl)amino)cyclobutane-1-carboxylate 6c:

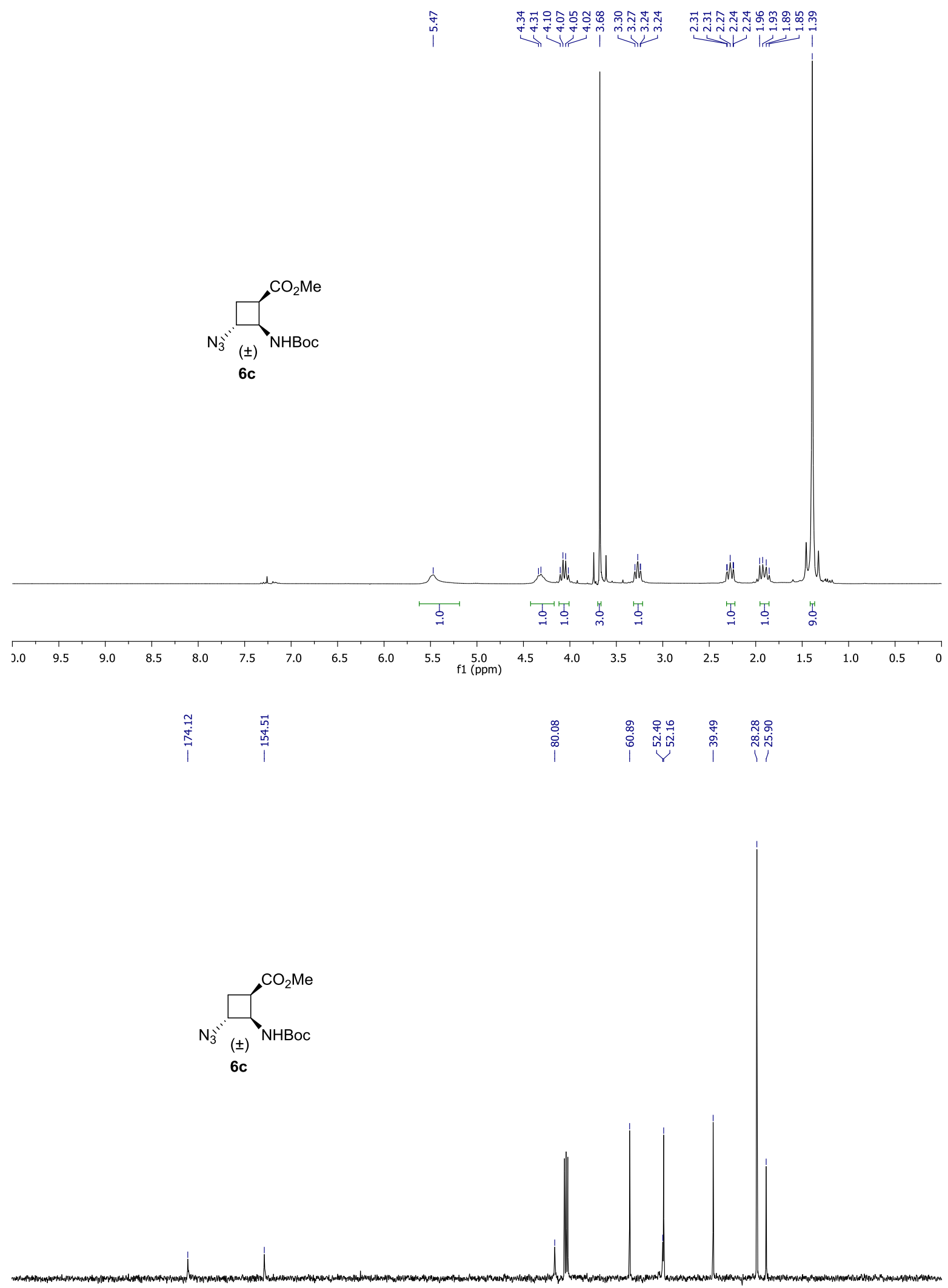

$\begin{array}{lllllllllllllllllllllllllllll}210 & 200 & 190 & 180 & 170 & 160 & 150 & 140 & 130 & 120 & 110 & 100 & 90 & 80 & 70 & 60 & 50 & 40 & 30 & 20 & 10 & 0 & -10\end{array}$ 
(士)-trans-trans-Methyl 3-azido-2-((tert-butoxycarbonyl)amino)cyclobutane-1-carboxylate 7c:
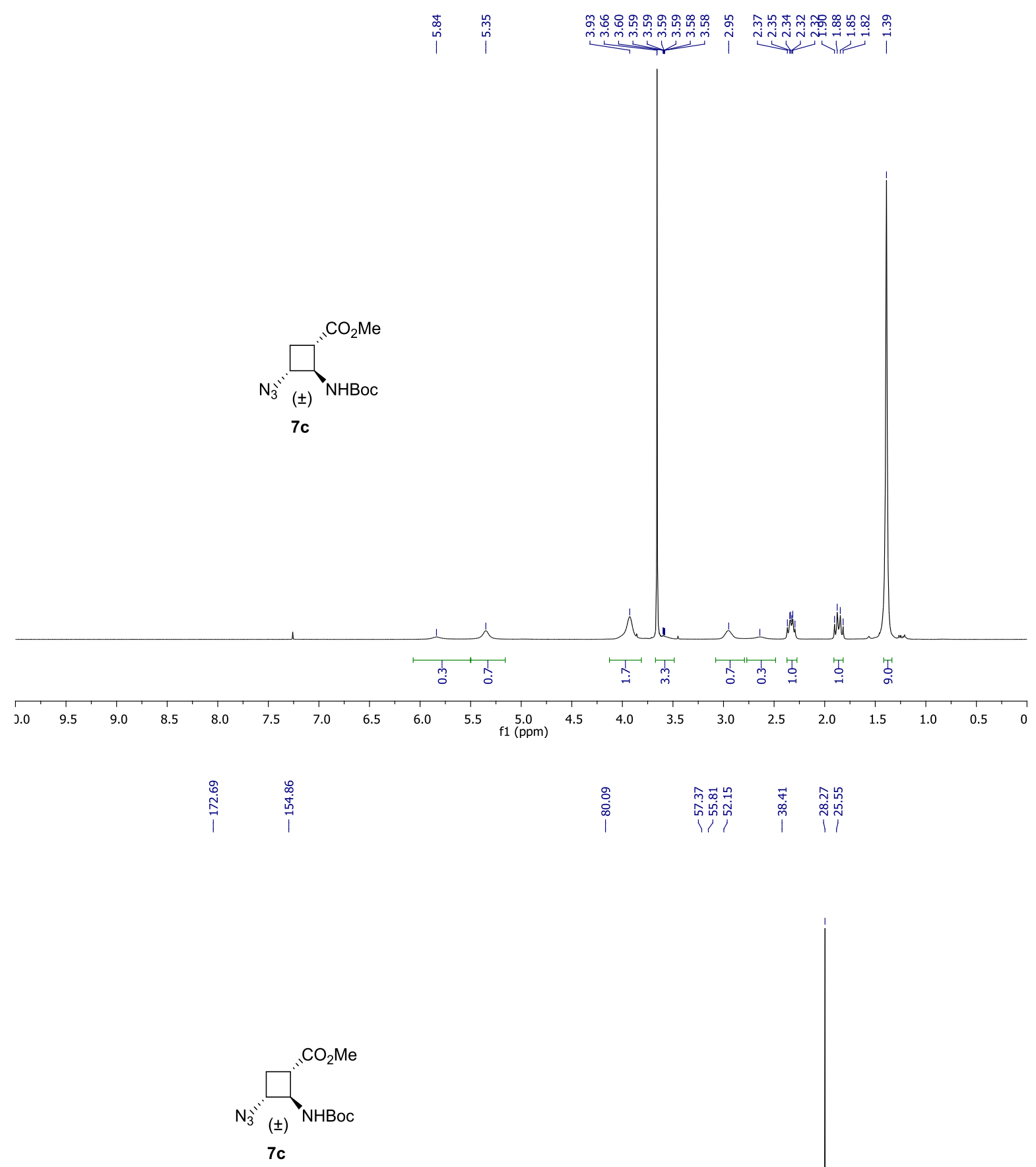

$7 c$

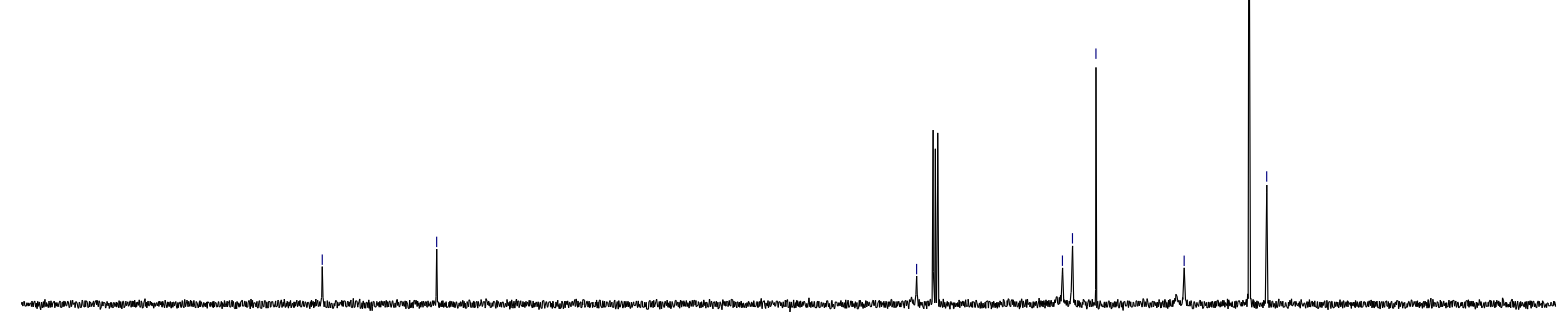

$\begin{array}{lllllllllllllllllllllllllllll}210 & 200 & 190 & 180 & 170 & 160 & 150 & 140 & 130 & 120 & 110 & 100 & 90 & 80 & 70 & 60 & 50 & 40 & 30 & 20 & 10 & 0 & -10\end{array}$ 
(士)-cis-trans-Methyl 2-((tert-butoxycarbonyl)amino)-3-hydroxycyclobutane-1-carboxylate 8a:

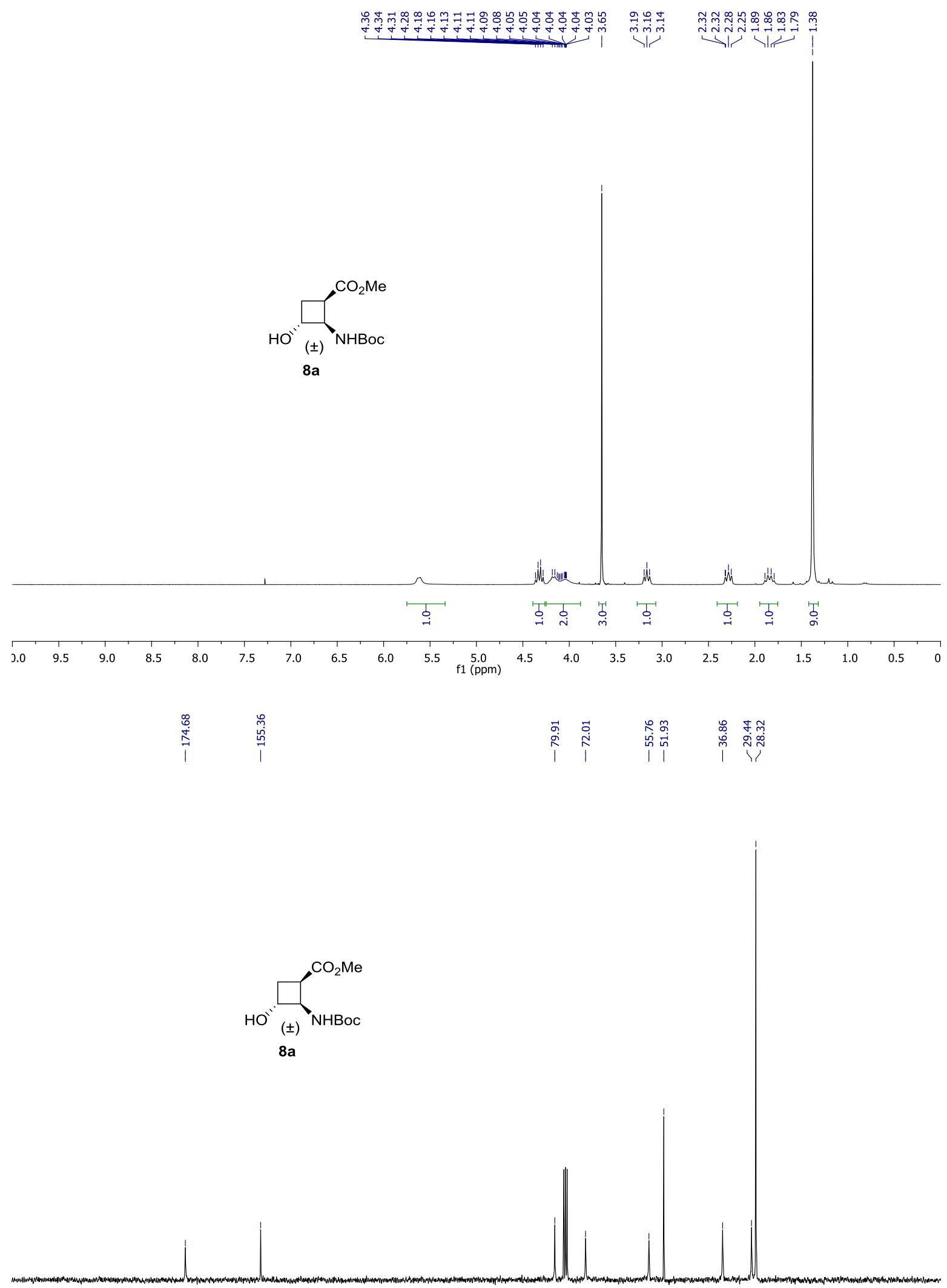

$\begin{array}{lllllllllllllllllllllllllllll}210 & 200 & 190 & 180 & 170 & 160 & 150 & 140 & 130 & 120 & 110 & \begin{array}{c}100 \\ \mathrm{f} 1(\mathrm{ppm})\end{array} & 90 & 80 & 70 & 60 & 50 & 40 & 30 & 20 & 10 & 0 & -10\end{array}$ 
(士)-trans-trans-Methyl 2-((tert-butoxycarbonyl)amino)-3-hydroxycyclobutane-1-carboxylate 9a:
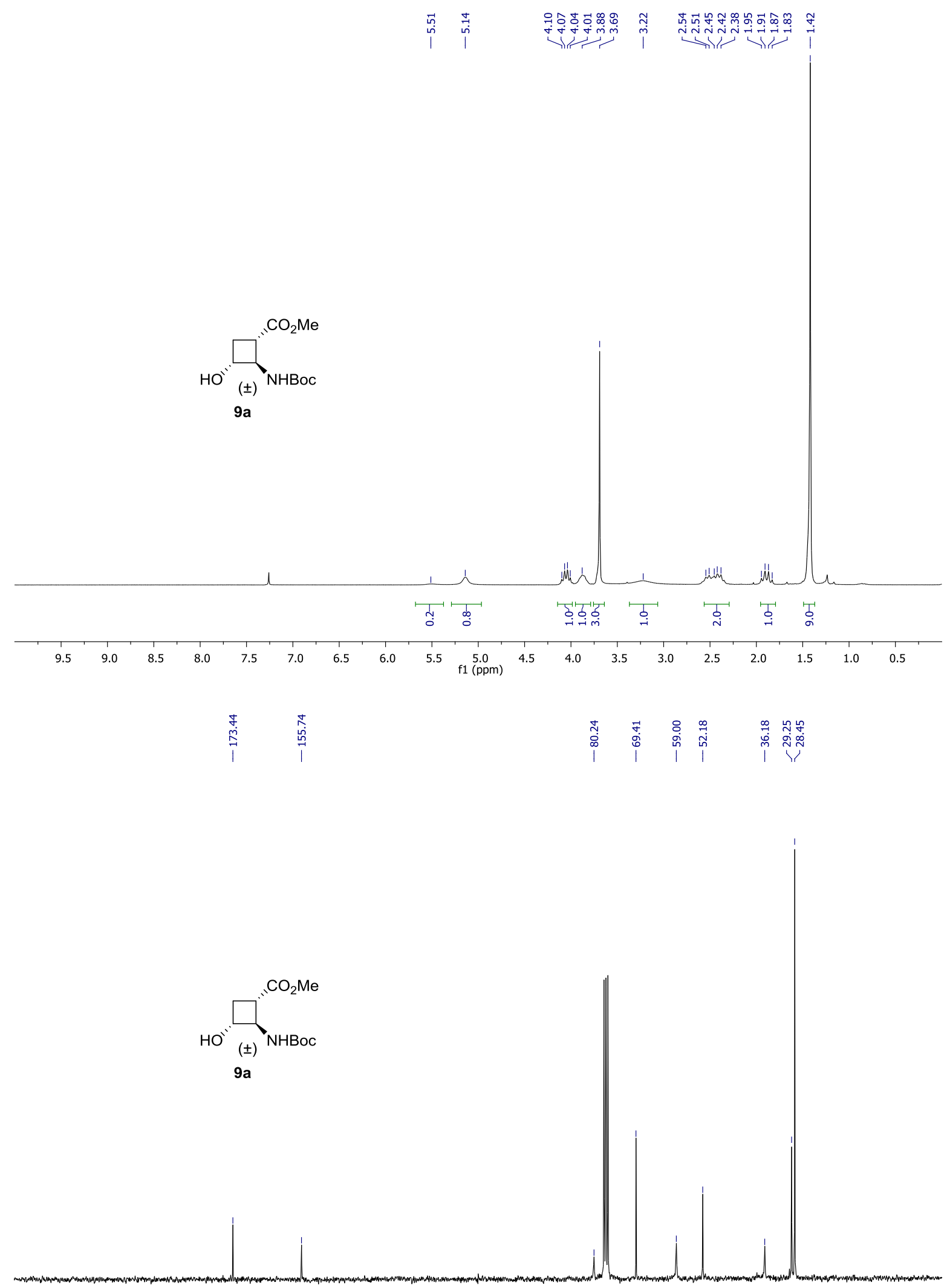

$\begin{array}{lllllllllllllllllllllllllllll}220 & 210 & 200 & 190 & 180 & 170 & 160 & 150 & 140 & 130 & 120 & 110 & 100 & 90 & 80 & 70 & 60 & 50 & 40 & 30 & 20 & 10 & 0 & 0\end{array}$ 
(士)-cis-trans-Methyl 2-((tert-butoxycarbonyl)amino)-3-mercaptocyclobutane-1-carboxylate 8b:
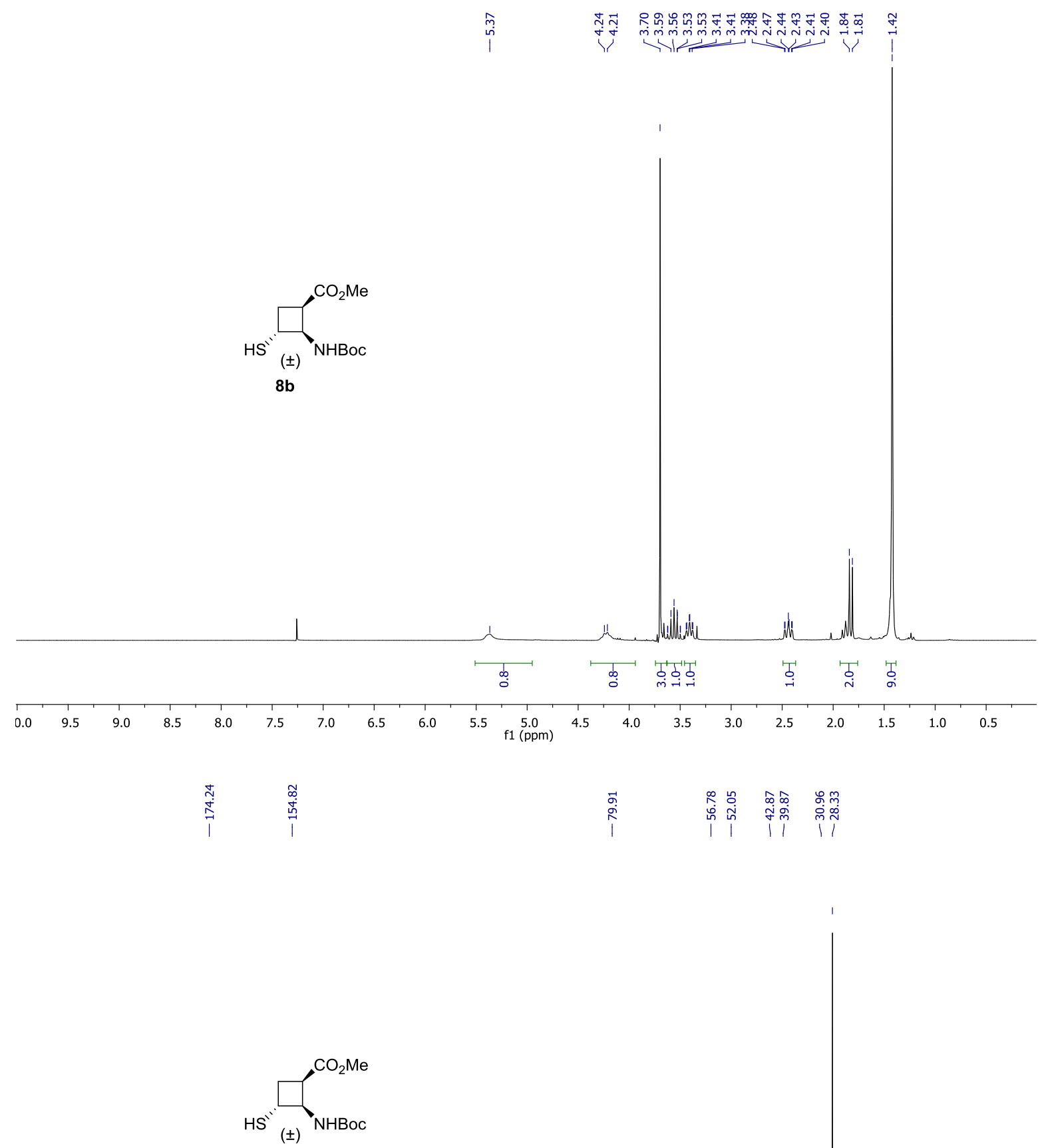

$8 \mathbf{b}$

$\begin{array}{lllllllllllllllllllllllllll}210 & 200 & 190 & 180 & 170 & 160 & 150 & 140 & 130 & 120 & 110 & \begin{array}{c}100 \\ \mathrm{f} 1(\mathrm{ppm})\end{array} & 90 & 80 & 70 & 60 & 50 & 40 & 30 & 20 & 10 & 0 & -10\end{array}$ 
(士)-trans-trans-Methyl 2-((tert-butoxycarbonyl)amino)-3-mercaptocyclobutane-1-carboxylate 9b:

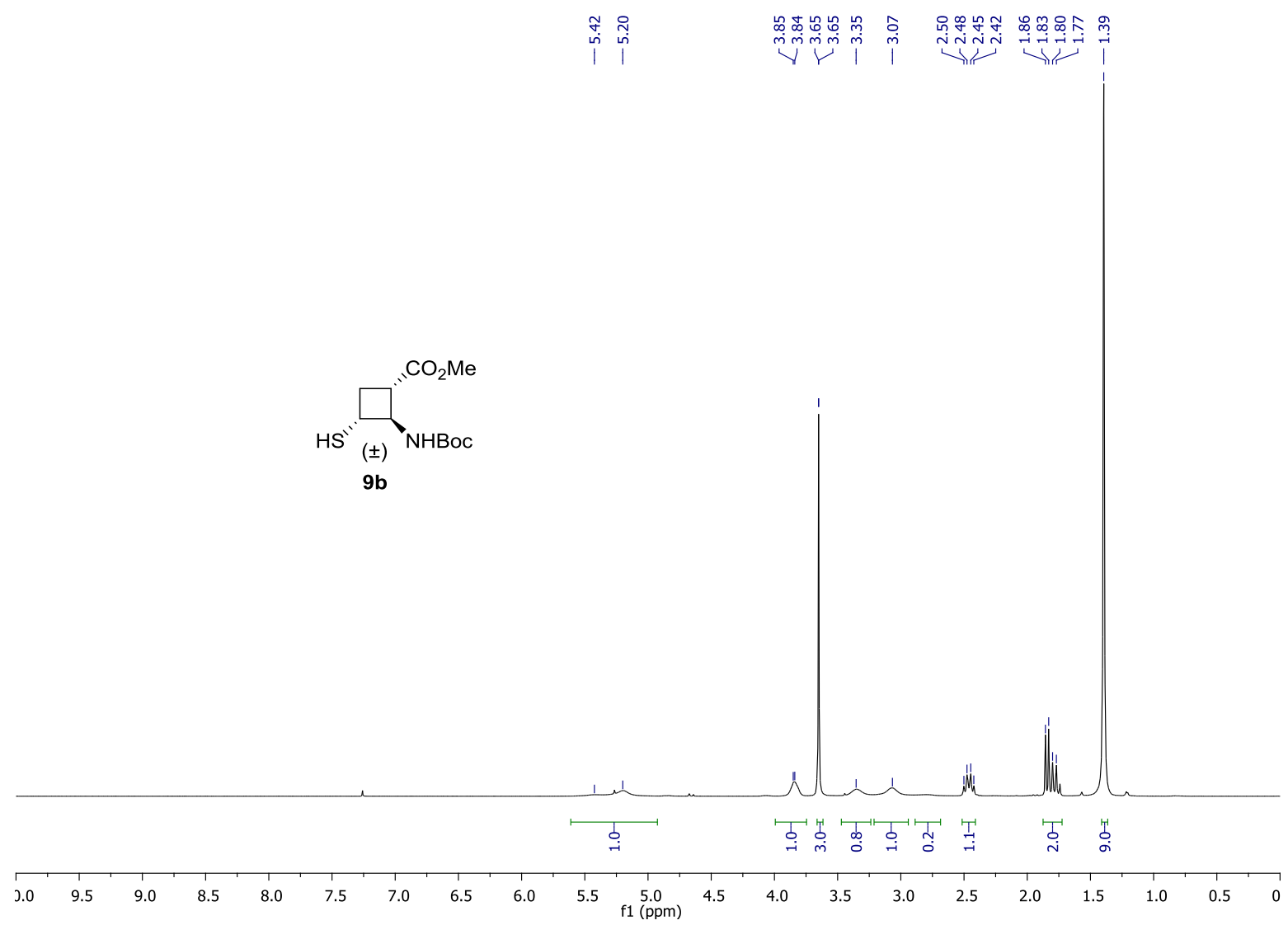

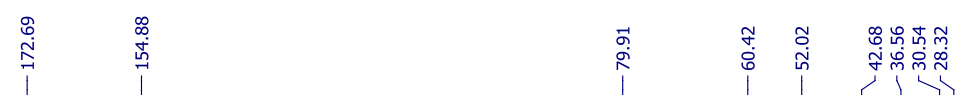

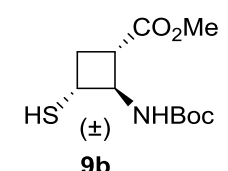

$9 b$

$\begin{array}{lllllllllllllllllllllll}210 & 200 & 190 & 180 & 170 & 160 & 150 & 140 & 130 & 120 & 110 & 100 & 90 & 80 & 70 & 60 & 50 & 40 & 30 & 20 & 10 & 0 & -10\end{array}$ 
(士)-cis-trans-Methyl 3-amino-2-((tert-butoxycarbonyl)amino)cyclobutane-1-carboxylate 8c:

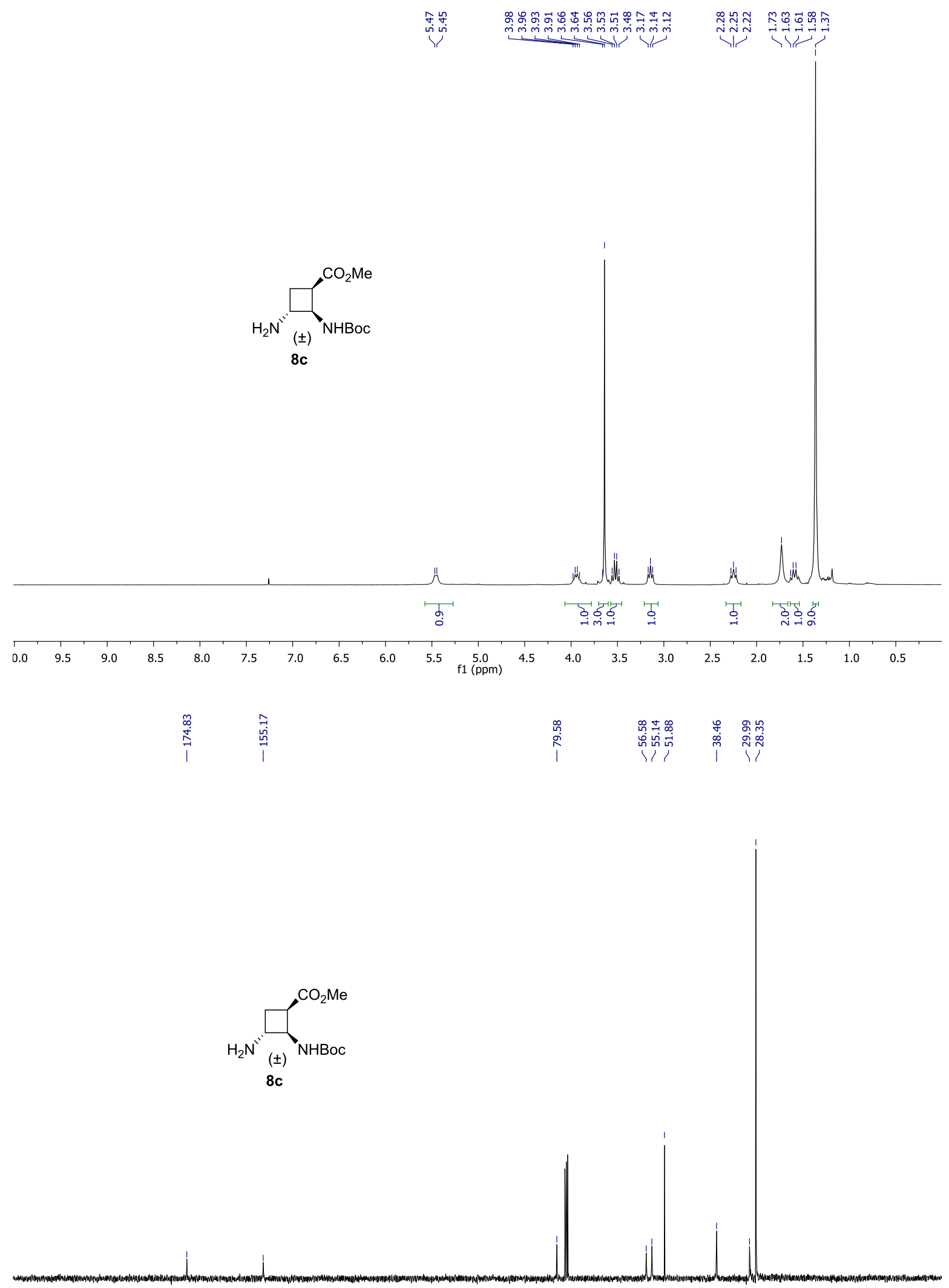

$\begin{array}{lllllllllllllllllllllll}210 & 200 & 190 & 180 & 170 & 160 & 150 & 140 & 130 & 120 & 110 & \begin{array}{c}100 \\ \mathrm{f} 1(\mathrm{ppm})\end{array} & 90 & 80 & 70 & 60 & 50 & 40 & 30 & 20 & 10 & 0 & -10\end{array}$ 
(士)-trans-trans-Methyl 3-amino-2-((tert-butoxycarbonyl)amino)cyclobutane-1-carboxylate 9c:

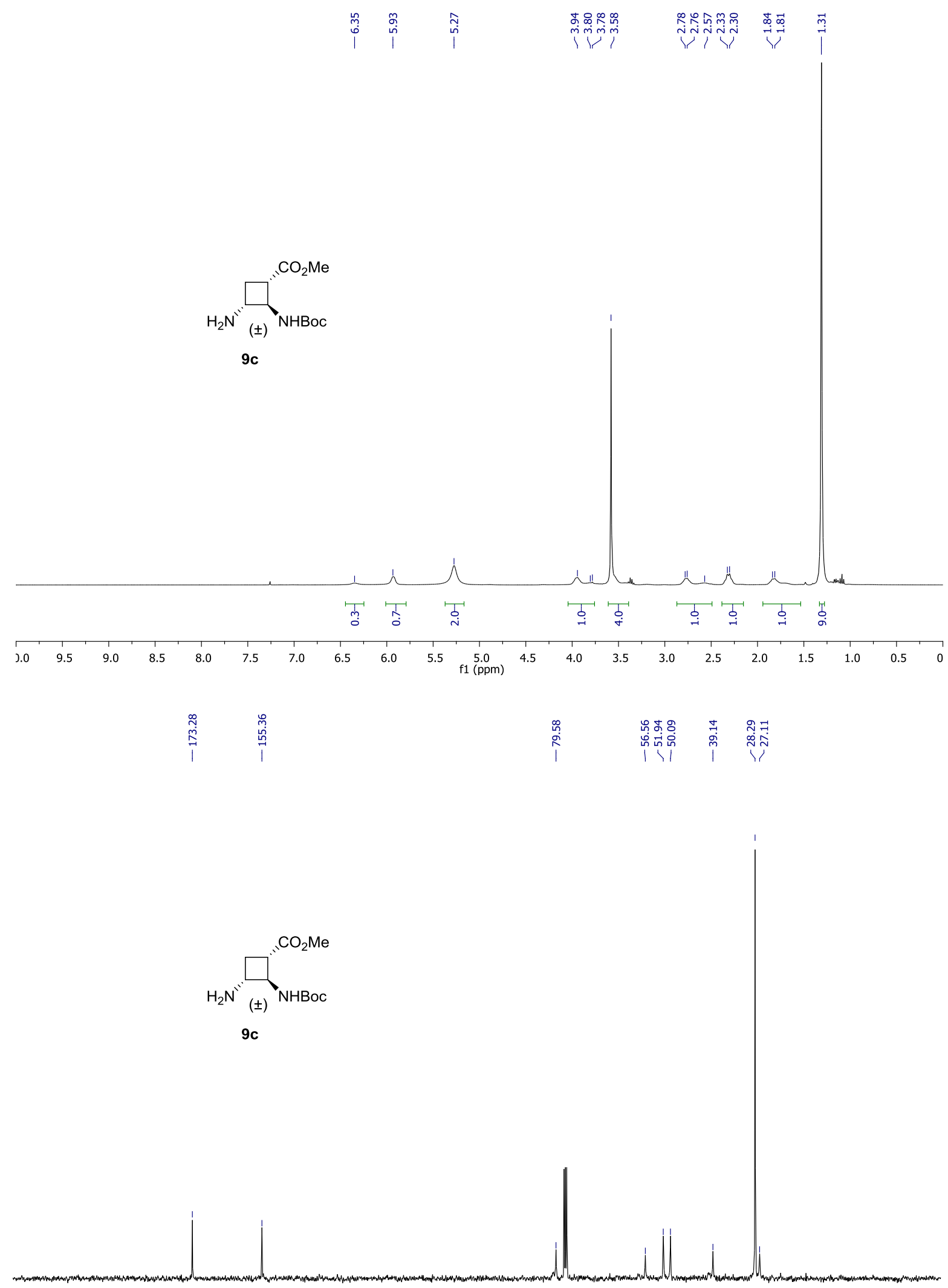

$\begin{array}{lllllllllllllllllllllll}210 & 200 & 190 & 180 & 170 & 160 & 150 & 140 & 130 & 120 & 110 & 100 & 90 & 80 & 70 & 60 & 50 & 40 & 30 & 20 & 10 & 0 & -10\end{array}$ 
(士)-cis-trans-2-Amino-3-hydroxycyclobutane-1-carboxylic acid 10a:

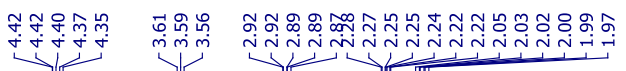
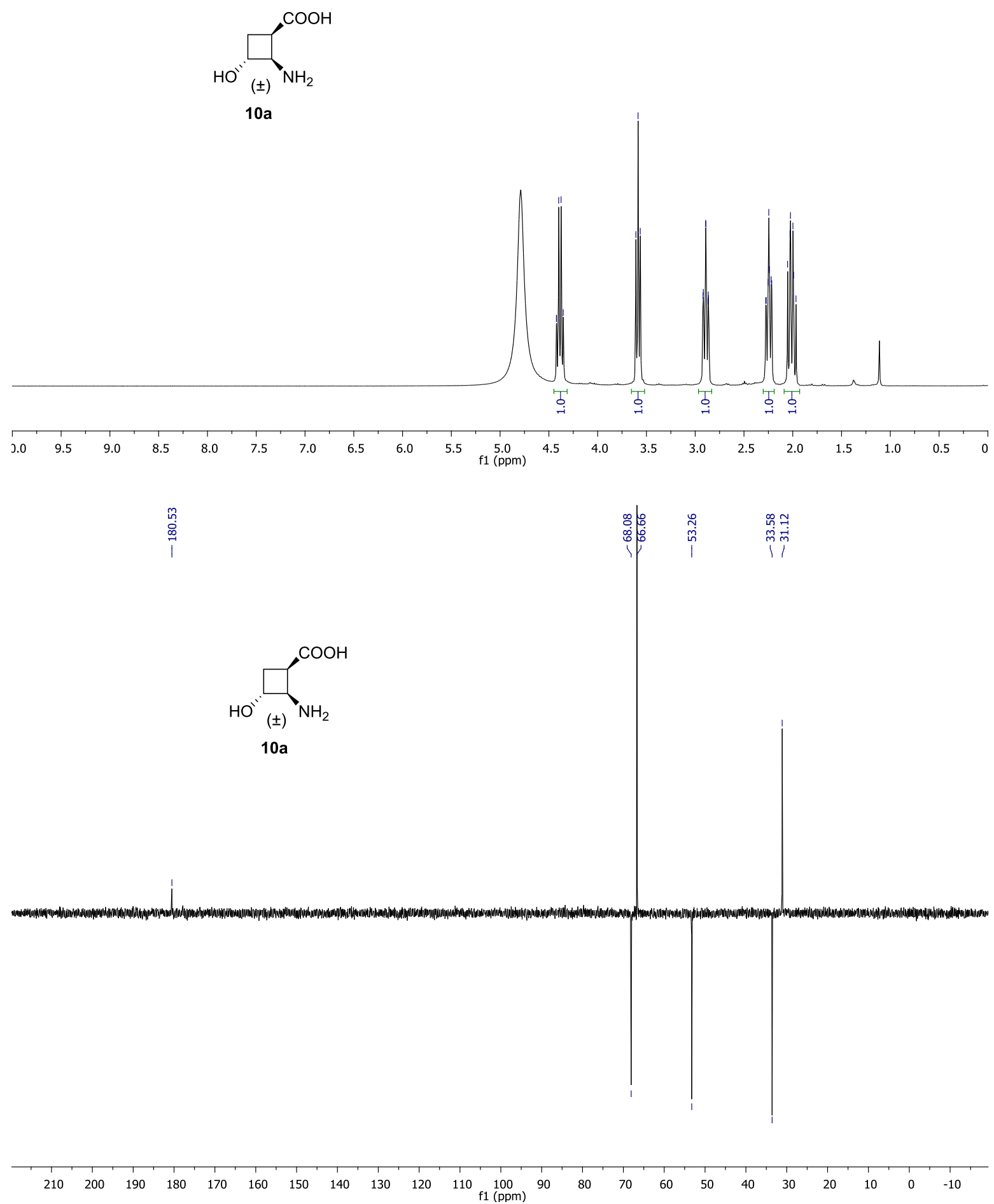
(士)-trans-trans-2-Amino-3-hydroxycyclobutane-1-carboxylic acid 11a:
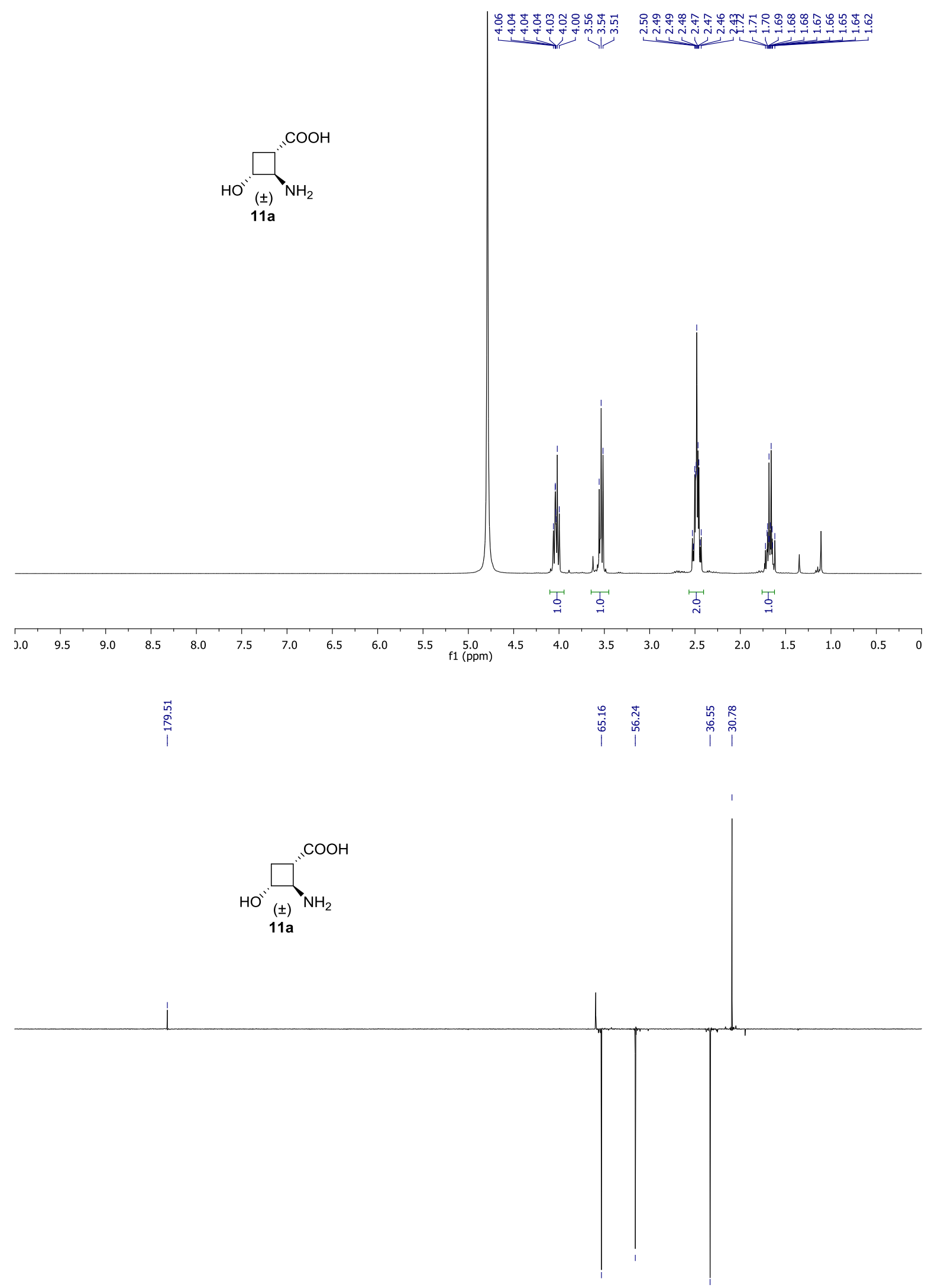

$\begin{array}{lllllllllllllllllllllllllllllllllll}210 & 200 & 190 & 180 & 170 & 160 & 150 & 140 & 130 & 120 & 110 & 100 & 90 & 80 & 70 & 60 & 50 & 40 & 30 & 20 & 10 & 0 & -10\end{array}$ 
(士)-cis-trans-2,3-Diaminocyclobutane-1-carboxylic acid 10c:
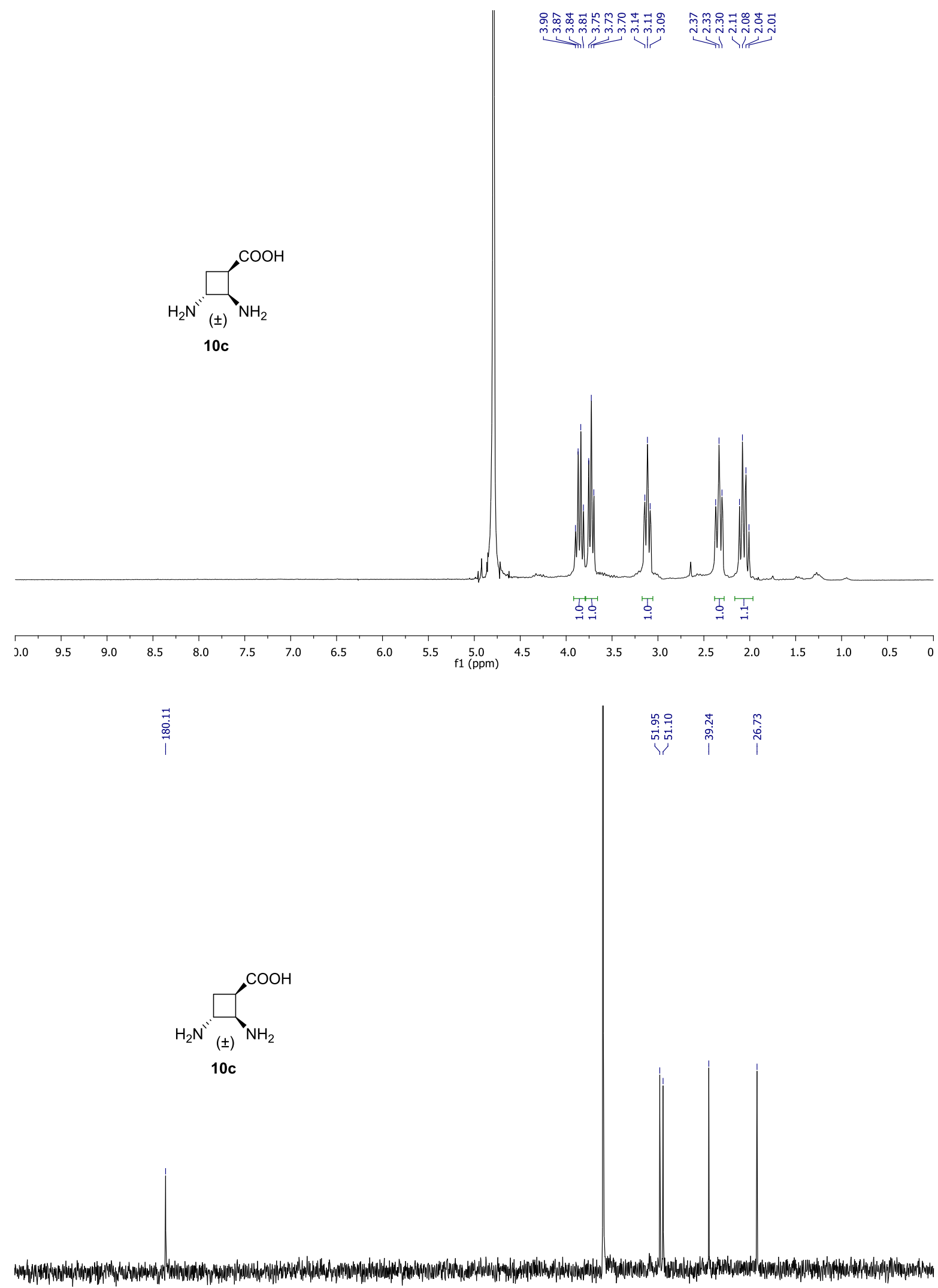

$\begin{array}{llllllllllllllllllllllllll}210 & 200 & 190 & 180 & 170 & 160 & 150 & 140 & 130 & 120 & 110 & 100 & 90 & 80 & 70 & 60 & 50 & 40 & 30 & 20 & 10 & 0 & -10\end{array}$ 
(士)-trans-trans-2,3-Diaminocyclobutane-1-carboxylic acid 11c:
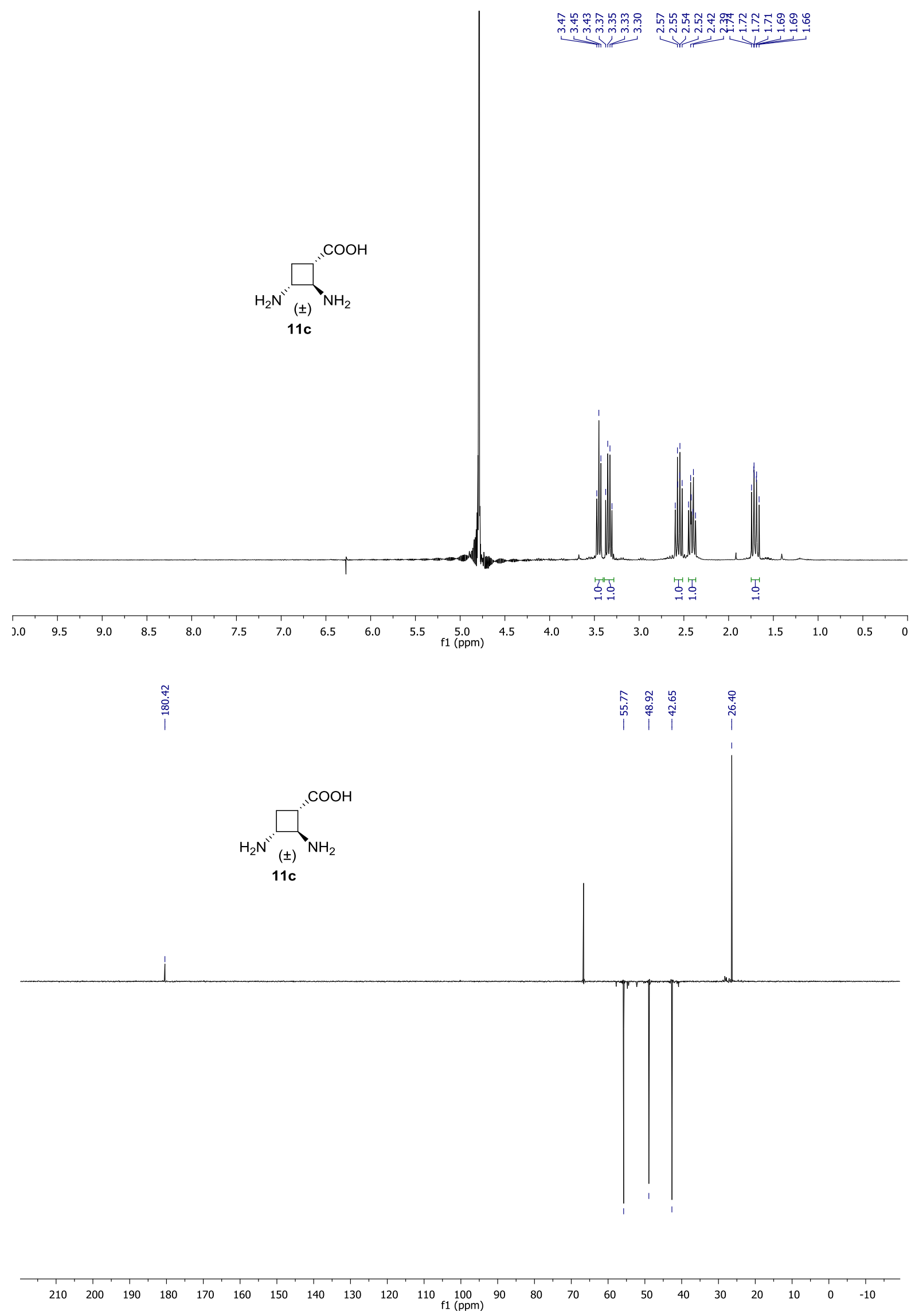
(士)-cis-trans-2-Amino-3-azidocyclobutane-1-carboxylic acid 10d:
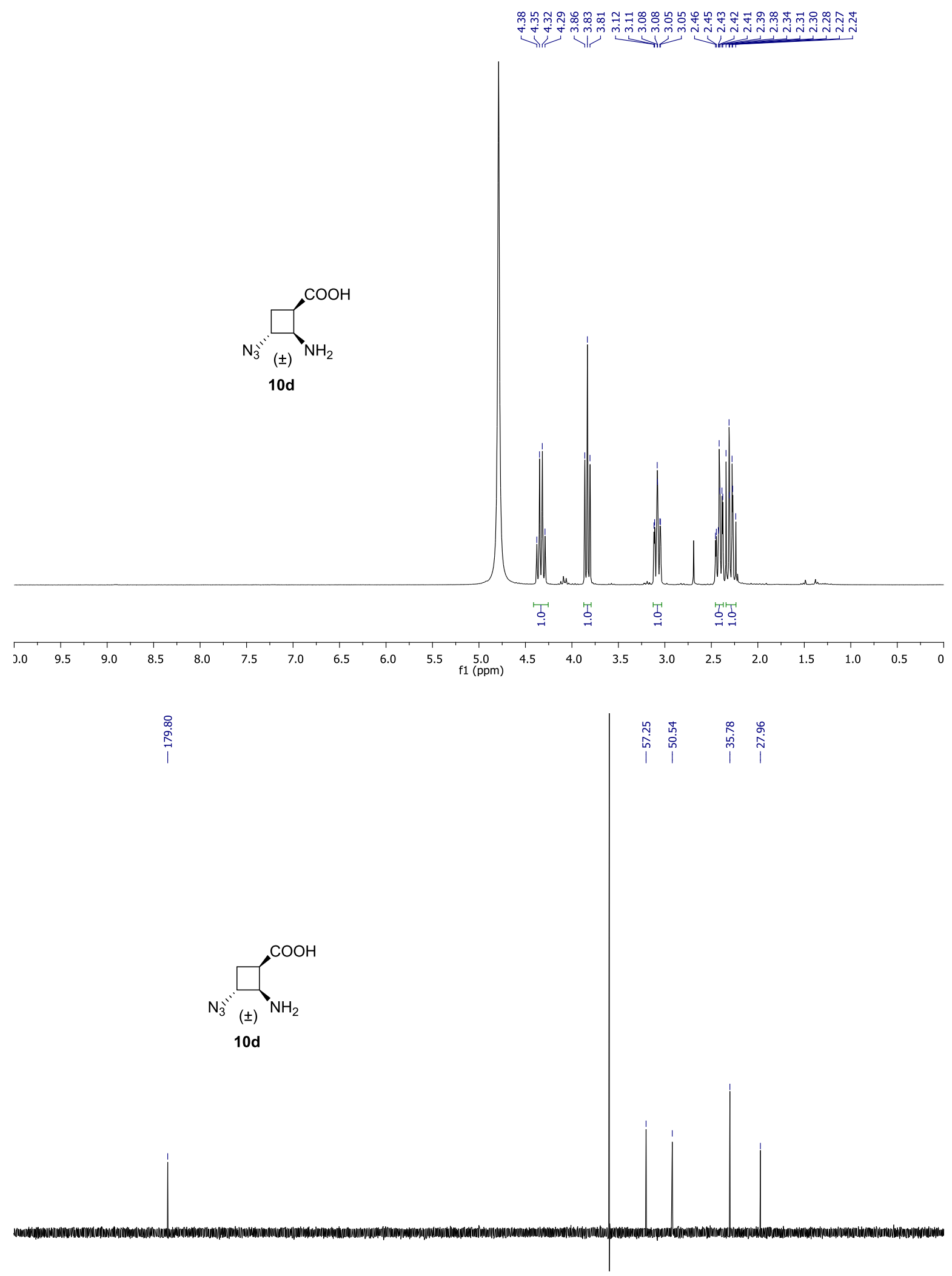

$\begin{array}{lllllllllllllllllllllllllllll}210 & 200 & 190 & 180 & 170 & 160 & 150 & 140 & 130 & 120 & 110 & 100 & 90 & 80 & 70 & 60 & 50 & 40 & 30 & 20 & 10 & 0 & -10\end{array}$ 
(士)-trans-trans-2-Amino-3-azidocyclobutane-1-carboxylic acid 11d:
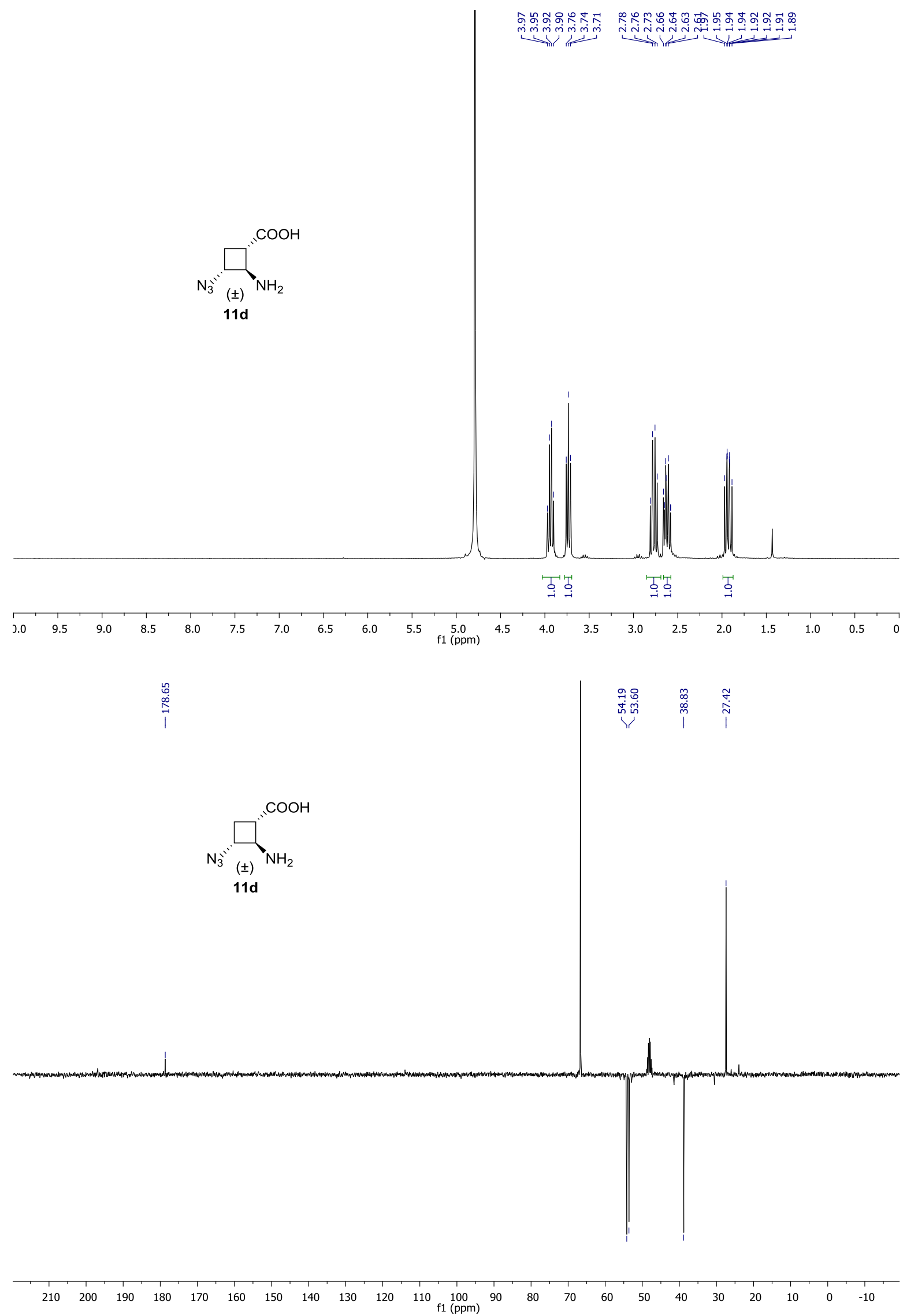
(士)-trans-cis-2-amino-3-hydroxycyclobutane-1-carboxylic acid 12a :
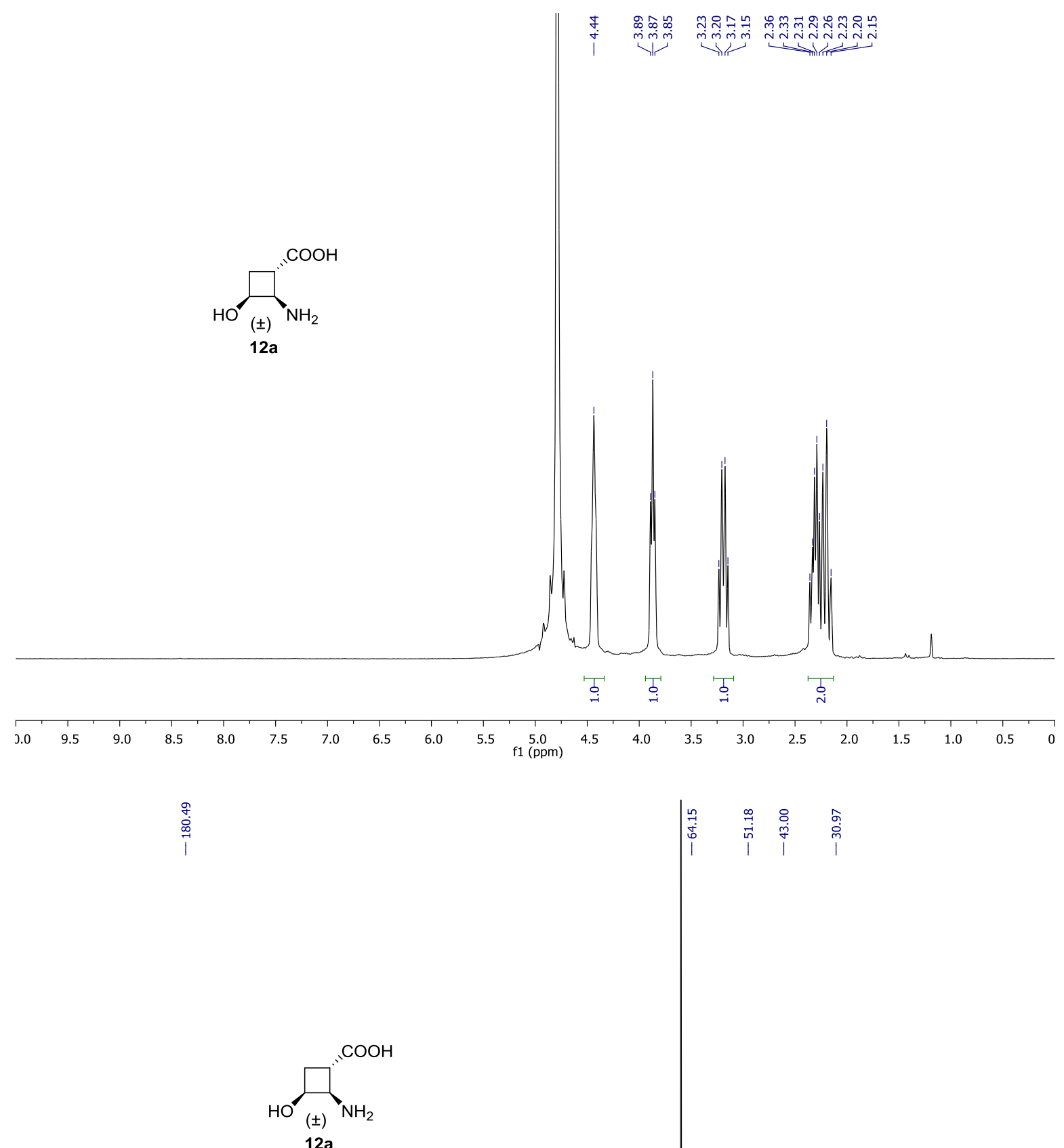

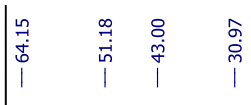

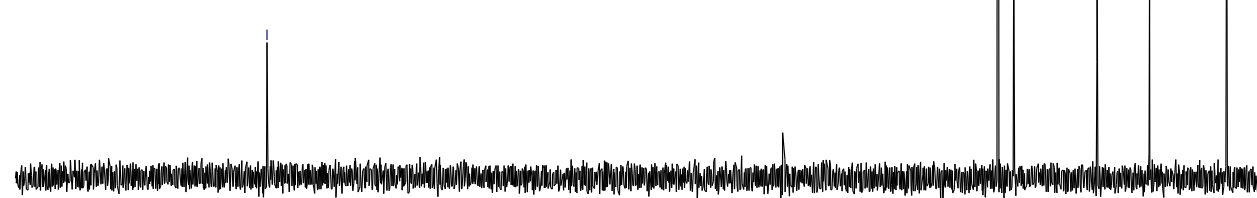

$\begin{array}{lllllllllllllllllllllll}210 & 200 & 190 & 180 & 170 & 160 & 150 & 140 & 130 & 120 & 110 & 100 & 90 & 80 & 70 & 60 & 50 & 40 & 30 & 20 & 10 & 0 & -10\end{array}$ 
(士)-cis-trans-Methyl 2-((tert-butoxycarbonyl)amino)-3-iodocyclobutane-1-carboxylate 14e:
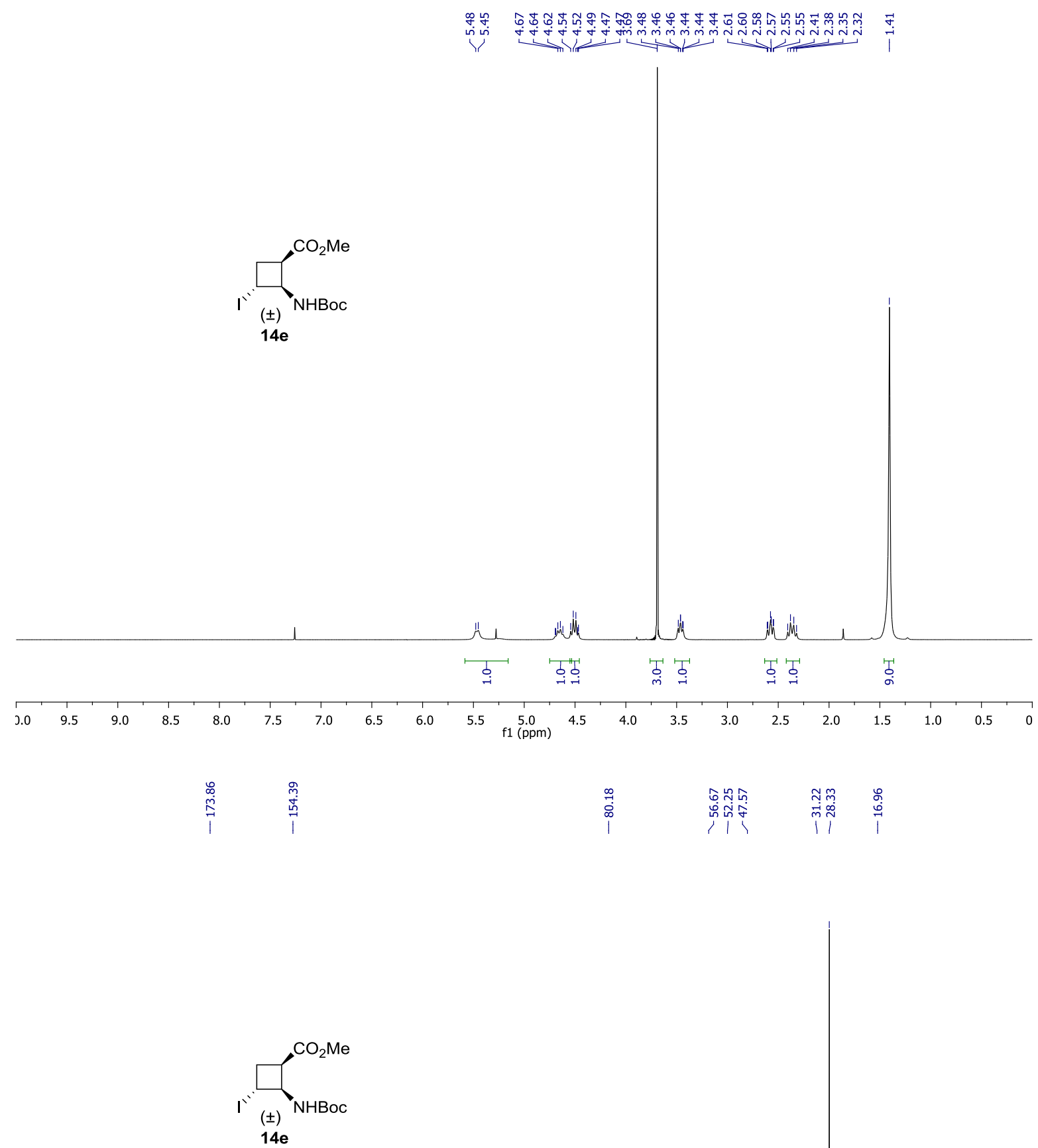

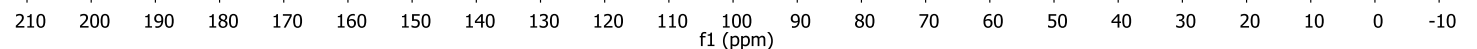


(士)-trans-trans-Methyl 2-((tert-butoxycarbonyl)amino)-3-iodocyclobutane-1-carboxylate 15e:

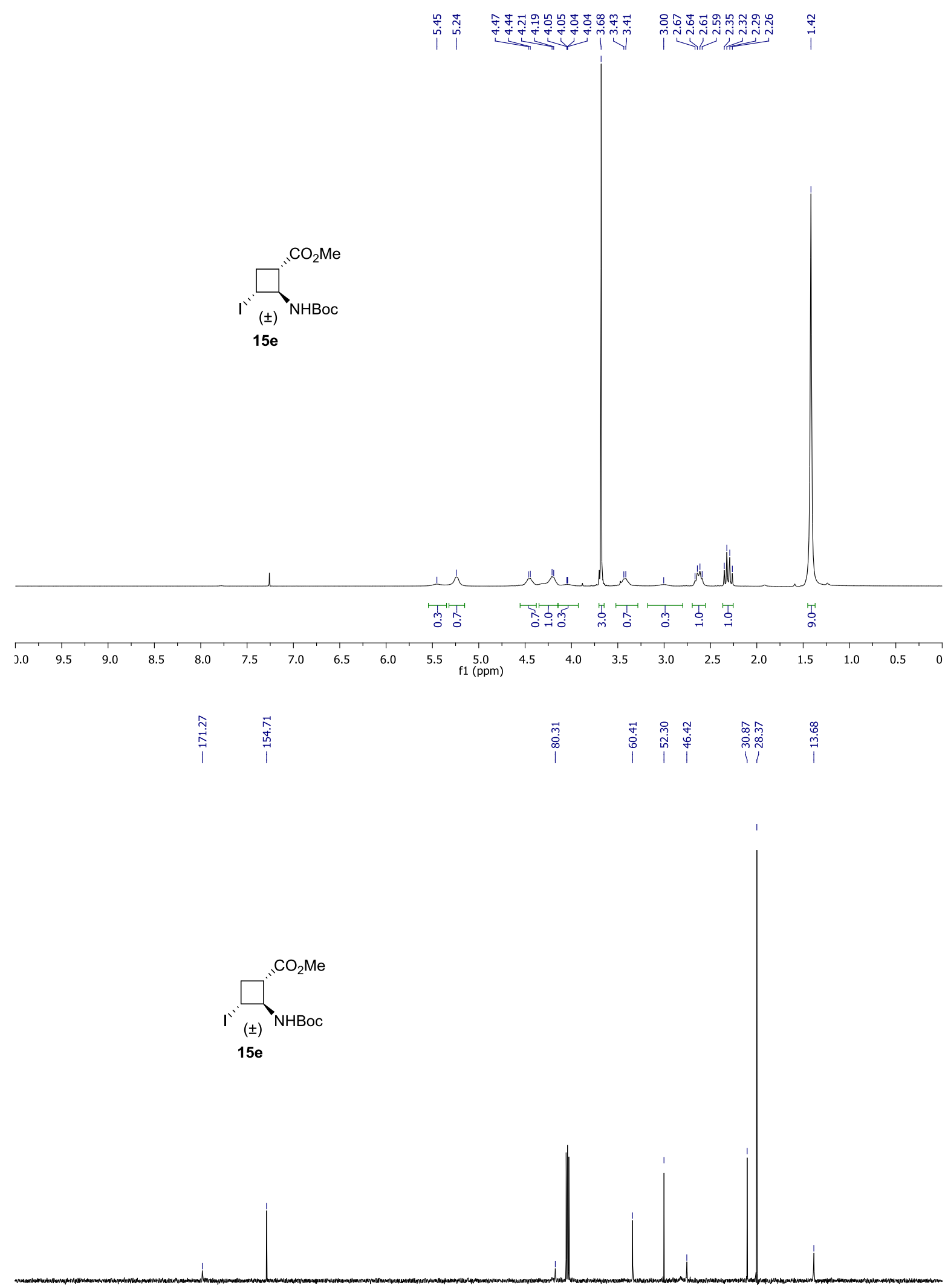

$\begin{array}{lllllllllllllllllllllll}210 & 200 & 190 & 180 & 170 & 160 & 150 & 140 & 130 & 120 & 110 & \begin{array}{c}100 \\ \mathrm{f} 1(\mathrm{ppm})\end{array} & 90 & 80 & 70 & 60 & 50 & 40 & 30 & 20 & 10 & 0 & -10\end{array}$ 
(士)-cis-trans-Methyl 3-bromo-2-((tert-butoxycarbonyl)amino)cyclobutane-1-carboxylate 14f:
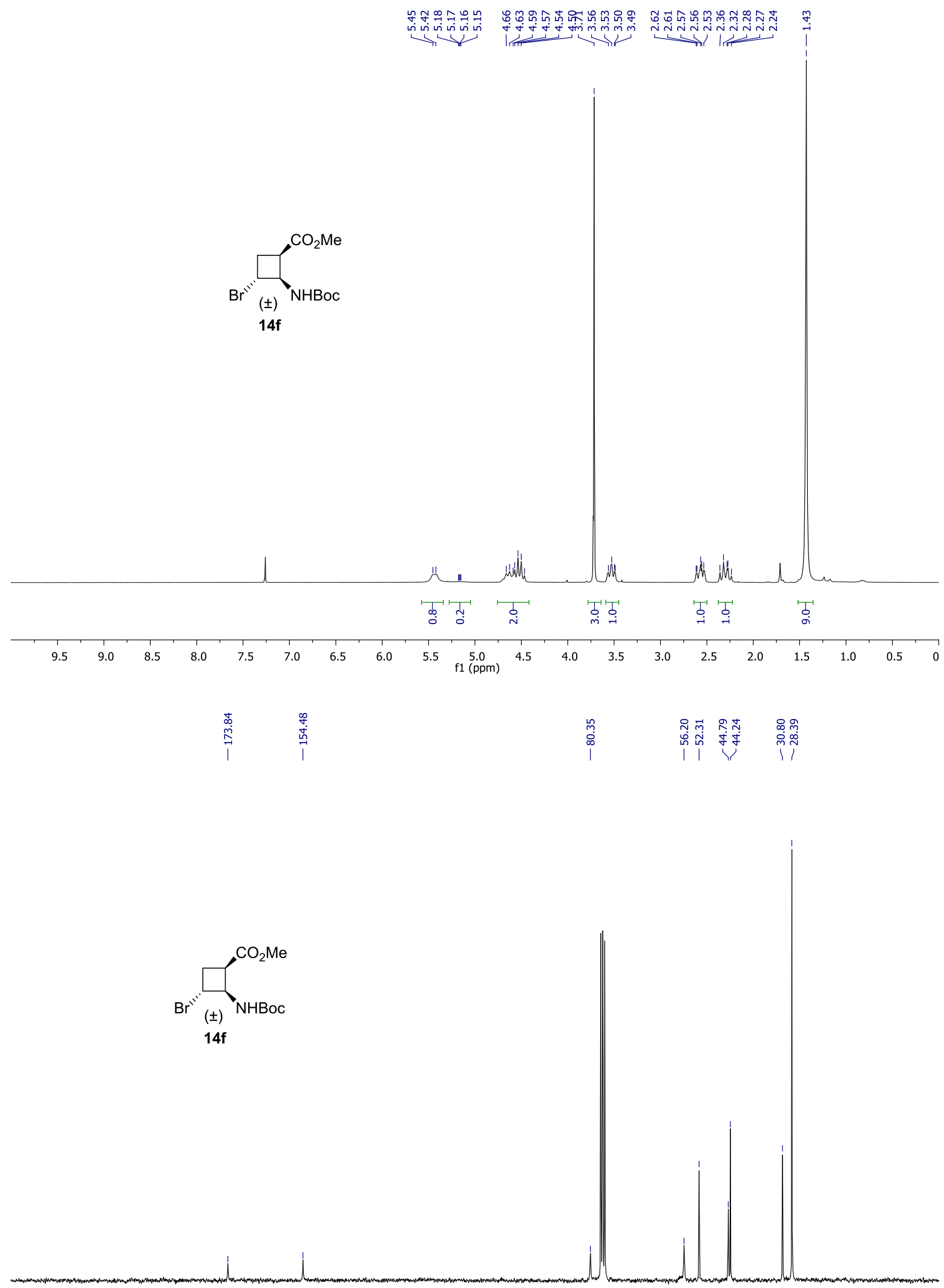

$\begin{array}{lllllllllllllllllllllll}220 & 210 & 200 & 190 & 180 & 170 & 160 & 150 & 140 & 130 & 120 & 110 & 100 & 90 & 80 & 70 & 60 & 50 & 40 & 30 & 20 & 10 & 0\end{array}$ 
(士)-trans-trans-Methyl 3-bromo-2-((tert-butoxycarbonyl)amino)cyclobutane-1-carboxylate 15f:

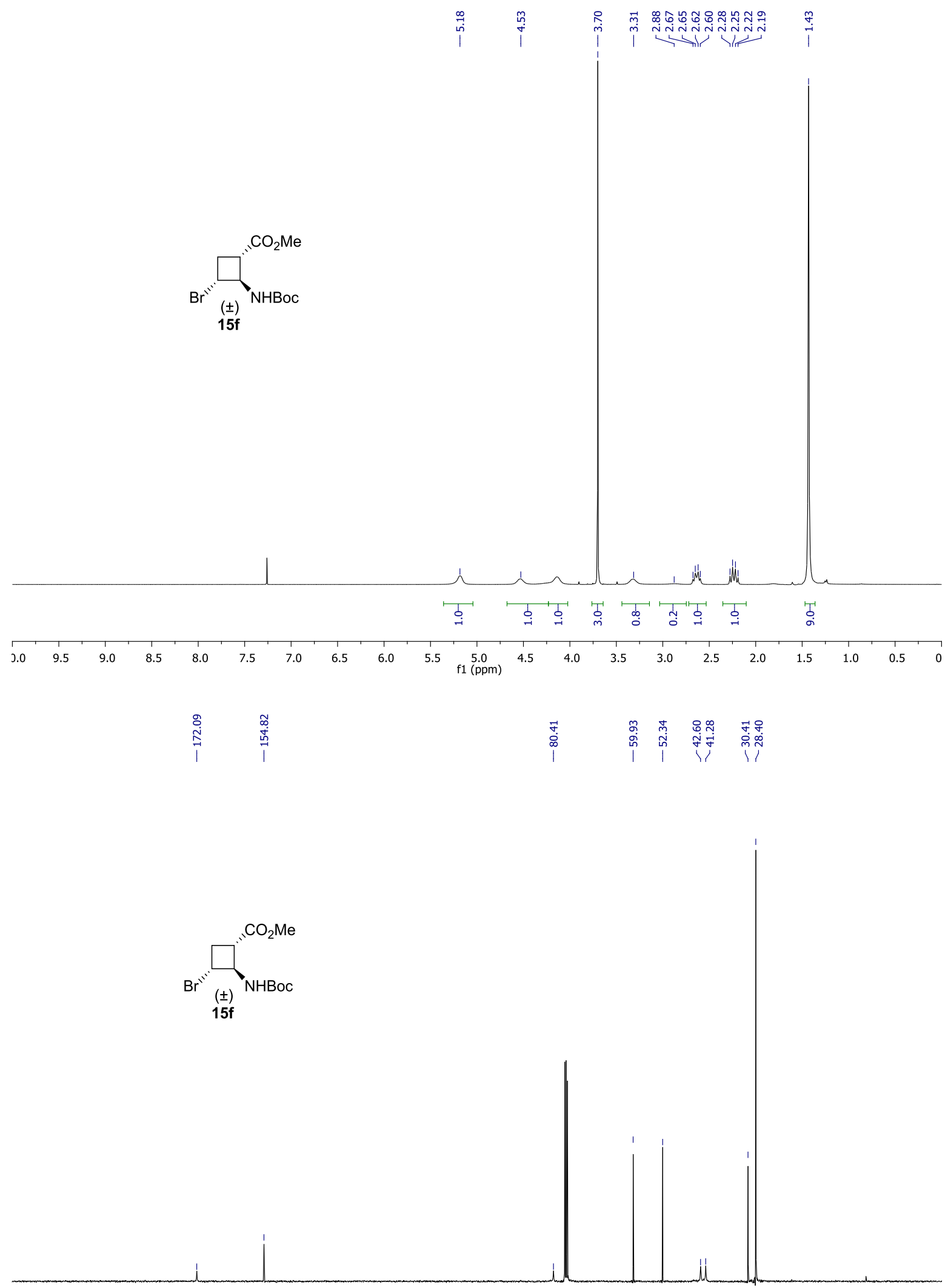

$\begin{array}{lllllllllllllllllllllll}210 & 200 & 190 & 180 & 170 & 160 & 150 & 140 & 130 & 120 & 110 & 100 & 90 & 80 & 70 & 60 & 50 & 40 & 30 & 20 & 10 & 0 & -10\end{array}$ 


\section{X-ray structure analysis of compounds $6 a$ and $7 a$}

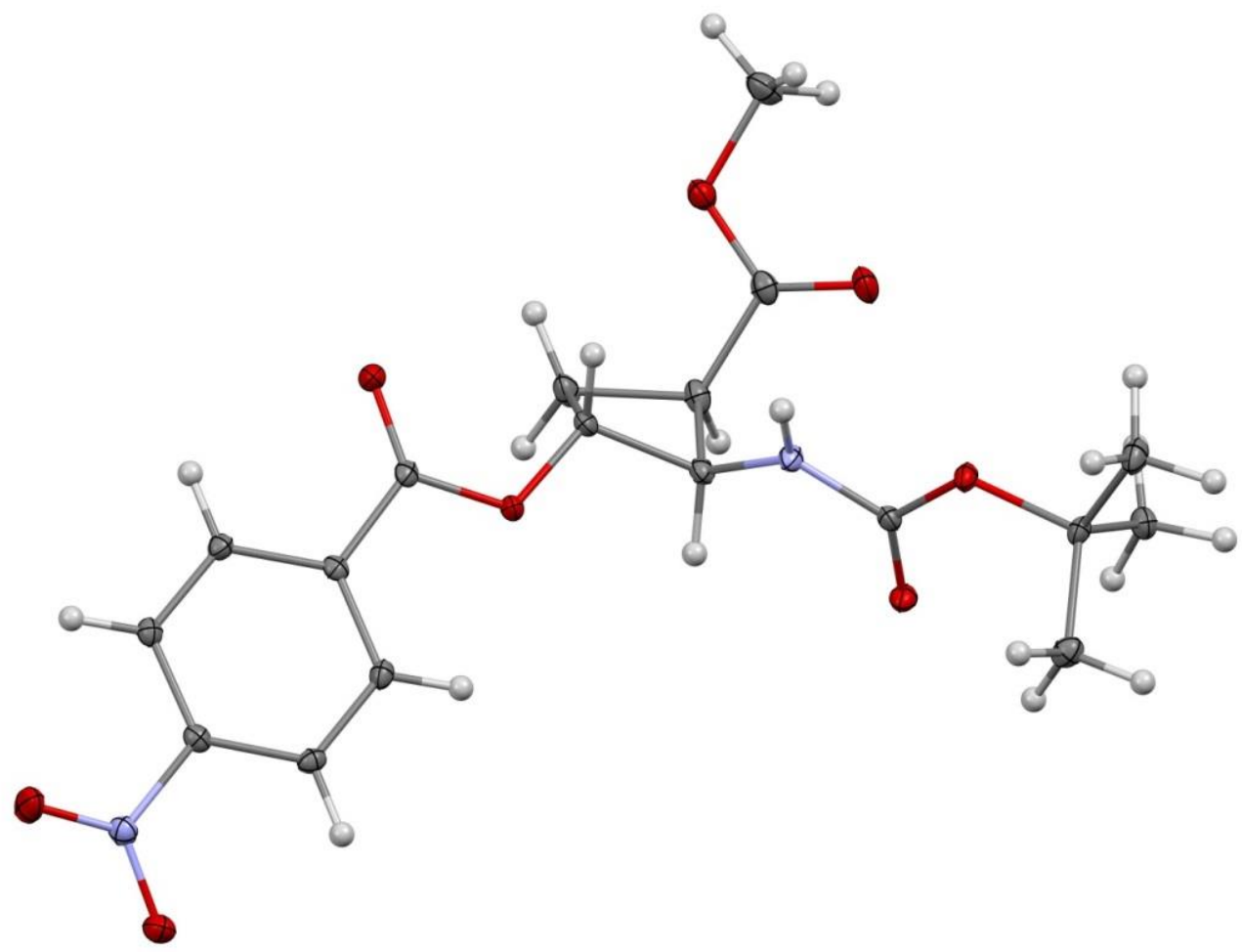

Fig. S1. An ORTEP drawing of compound ( \pm )-6a (XRay1). Thermal ellipsoids are shown at the $30 \%$ level. The crystal was grown from a solution in diethyl ether by vapor diffusion of pentane.

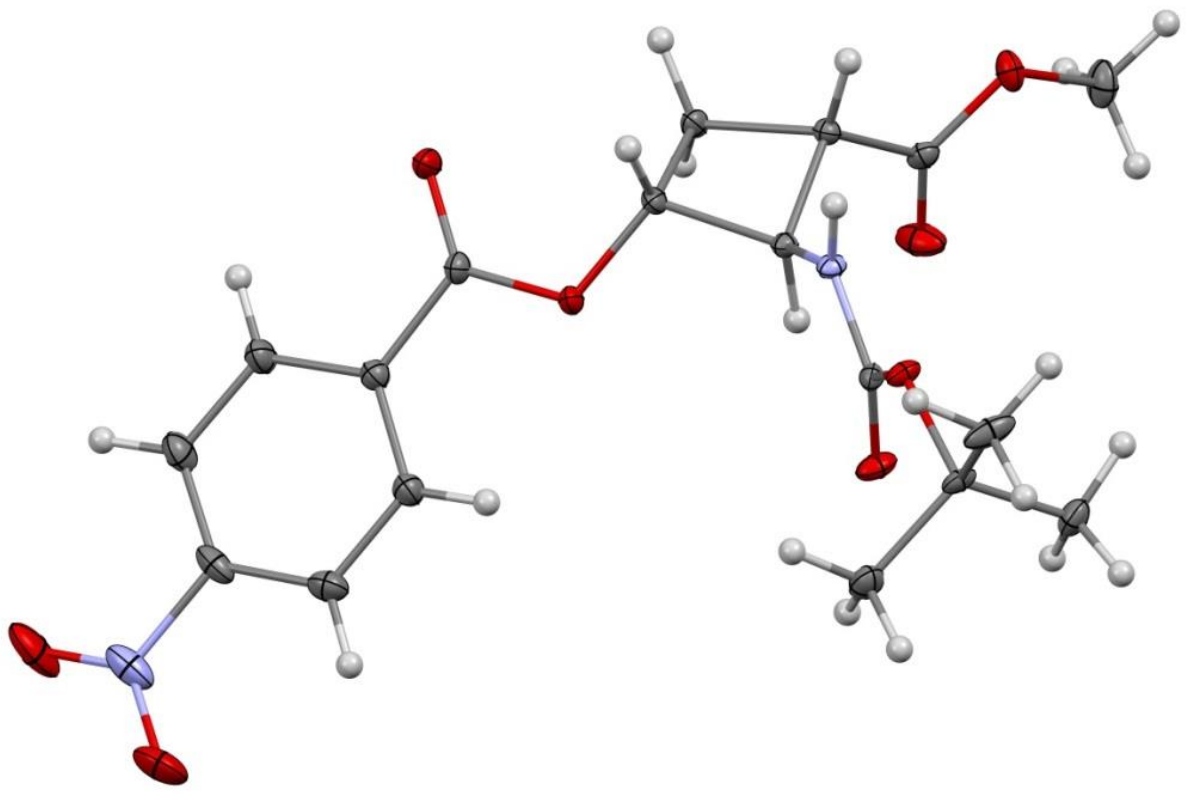

Fig. S2. An ORTEP drawing of compound ( \pm )-7a (XRay2). Thermal ellipsoids are shown at the $30 \%$ level (one molecule of the asymmetric unit, solvent are omitted for clarity)

The crystal was grown from a solution in ethyl acetate by vapor diffusion of pentane. 
X-ray diffraction data for compounds 6a (XRay1) and 7a (XRay2) were collected by using a VENTURE PHOTON100 CMOS Bruker diffractometer with Micro-focus luS source CuKa radiation. Crystals were mounted on a CryoLoop (Hampton Research) with Paratone-N (Hampton Research) as cryoprotectant and then flash-frozen in a nitrogen gas stream at $100 \mathrm{~K}$. For compounds, the temperature of the crystal was maintained at the selected value by means of a $\mathrm{N}$-Helix cooling device to within an accuracy of $\pm 1 \mathrm{~K}$. The data were corrected for Lorentz polarization and absorption effects. The structures were solved by direct methods using SHELXS-97 $7^{\text {S1 }}$ and refined against $F^{2}$ by full-matrix least-squares techniques using SHELXL$2018^{\mathrm{S2}}$ with anisotropic displacement parameters for all non-hydrogen atoms. Hydrogen atoms were located on a difference Fourier map and introduced into the calculations as a riding model with isotropic thermal parameters. All calculations were performed by using the Crystal Structure crystallographic software package WINGX. ${ }^{53}$

The crystal data collection and refinement parameters are given in Table S2.

CCDC 1898946 (6a:XRay1) \& 1898947 (7a:XRay2) contains the supplementary crystallographic data for this paper. These data can be obtained free of charge from the Cambridge Crystallographic Data Centre via http://www.ccdc.cam.ac.uk/Community/Requestastructure.

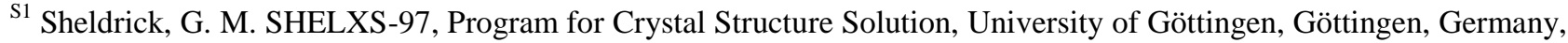
1997.

${ }^{\text {s2 }}$ Sheldrick, G. M. A short history of SHELX. Acta Cryst. A 2008, 64, 112-122.

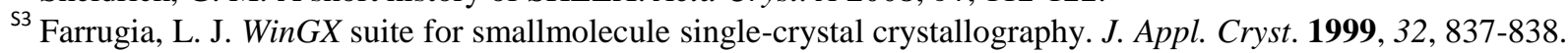


Table S2. Crystallographic data and structure refinement details.

\begin{tabular}{|c|c|c|}
\hline Compound & 6a (XRay1) & 7a (XRay2) \\
\hline $\mathrm{CCDC}$ & 1898946 & 1898947 \\
\hline Empirical Formula & $\mathrm{C}_{18} \mathrm{H}_{22} \mathrm{~N}_{2} \mathrm{O}_{8}$ & $\begin{array}{c}3\left(\mathrm{C}_{18} \mathrm{H}_{22} \mathrm{~N}_{2} \mathrm{O}_{8}\right),\left(\mathrm{C}_{4} \mathrm{H}_{8} \mathrm{O}_{2}\right), \\
0.5\left(\mathrm{C}_{4} \mathrm{O}_{2}\right)\end{array}$ \\
\hline$M_{r}$ & 394.37 & 1311.25 \\
\hline Crystal size, $\mathrm{mm}^{3}$ & $0.13 \times 0.04 \times 0.03$ & $0.23 \times 0.06 \times 0.02$ \\
\hline Crystal system & orthorhombic & monoclinic \\
\hline Space group & $P 2_{1} 2_{1} 2_{1}$ & $P 21 / c$ \\
\hline a, $\AA$ & $5.3084(3)$ & $13.6074(6)$ \\
\hline$b, \AA$ & $14.0286(8)$ & $15.3263(6)$ \\
\hline$c, \AA$ & $25.1623(13)$ & $32.0745(13)$ \\
\hline$\alpha,{ }^{\circ}$ & 90 & 90 \\
\hline$\beta,{ }^{\circ}$ & 90 & $92.113(2)$ \\
\hline$v_{1}^{\circ}$ & 90 & 90 \\
\hline Cell volume, $\AA^{3}$ & $1873.82(18)$ & $6684.6(5)$ \\
\hline$Z ; Z^{\prime}$ & $4 ; 1$ & $4 ; 1$ \\
\hline $\mathrm{T}, \mathrm{K}$ & $100(1)$ & $100(1)$ \\
\hline $\begin{array}{c}\text { Radiation type ; } \\
\text { wavelength } \AA\end{array}$ & CuK $\alpha ; 1.54178$ & CuK $\alpha ; 1.54178$ \\
\hline$F_{000}$ & 832 & 2768 \\
\hline$\mu, \mathrm{mm}^{-1}$ & 0.942 & 0.879 \\
\hline$\theta$ range $^{\circ}$ & $3.513-66.690$ & $2.757-70.240$ \\
\hline Reflection collected & 27592 & 116009 \\
\hline Reflections unique & 3328 & 12688 \\
\hline $\mathrm{R}_{\text {int }}$ & 0.0520 & 0.0745 \\
\hline GOF & 1.033 & 1.019 \\
\hline Refl. obs. $(1>2 \sigma(I))$ & 3163 & 9463 \\
\hline Parameters & 257 & 849 \\
\hline$w R_{2}$ (all data) & 0.0662 & 0.1495 \\
\hline $\mathrm{R}$ value $(I>2 \sigma(I))$ & 0.0271 & 0.0547 \\
\hline $\begin{array}{l}\text { Largest diff. peak and } \\
\text { hole }\left(\mathrm{e}-. \AA^{-3}\right)\end{array}$ & $0.314 ;-0.230$ & $0.730 ;-0.695$ \\
\hline
\end{tabular}

